\title{
Modelling the Dynamics of Endemic Malaria Disease with Imperfect Quarantine and Optimal Control
}

\author{
Dereje Gutema Edossa ${ }^{*}$, Alemu Geleta Wedajo, Purnachandra Rao Koya \\ Department of Mathematics, Wollega University, Nekemte, Ethiopia \\ Email address: \\ derejegutema8@gmail.com (D. G. Edossa),alemugeleta@gmail.com (A. G. Wedajo),drkpraophd@gmail.com (P. R. Koya) \\ ${ }^{*}$ Corresponding author
}

\section{To cite this article:}

Dereje Gutema Edossa, Alemu Geleta Wedajo, Purnachandra Rao Koya. Modelling the Dynamics of Endemic Malaria Disease with Imperfect Quarantine and Optimal Control. Mathematical Modelling and Applications. Vol. 6, No. 2, 2021, pp. 29-55.

doi: $10.11648 /$ j.mma.20210602.12

Received: May 20, 2021; Accepted: July 7, 2021; Published: July 16, 2021

\begin{abstract}
Malaria is an infectious disease caused by Plasmodium parasite and it is transmitted among humans through bites of female Anopheles mosquitoes. In this paper, a new deterministic mathematical model for the endemic malaria disease transmission that incorporates imperfect quarantine and optimal control is proposed. Impact of various intervention strategies in the community with varying population at time $t$ are analyzed using mathematical techniques. Further, the model is analyzed using stability theory of differential equations and the basic reproduction number is obtained from the largest eigenvalue of the next-generation matrix. Conditions for local and global stability of disease free, local stability of endemic equilibria and bifurcations are determined in terms of the basic reproduction number. The Center manifold theory is used to analyze the bifurcation of the model. It is shown that the model exhibit both a backward and a forward bifurcation. Reducing the biting rate of the quarantined people is advice able to minimize the spread of endemic malaria disease. The optimal control is designed by applying Pontryagins's Maximum Principle (PMP) with four control strategies namely, insecticide treated nets, screening, treatment and indoor residual spray. The best strategy to control endemic malaria disease is the combination that incorporated all four control strategies.
\end{abstract}

Keywords: Endemic Malaria, Imperfect Quarantine, Reproduction Number, Stability, Bifurcation, Optimal Control

\section{Introduction}

Malaria is the dangerous one among infectious disease. It is caused by plasmodium parasites that are transmitted among humans through the bites of female Anopheles mosquitoes. And it is also the largest burden disease for these people living in poor countries, especially, in Sub Saharan Africa, causing high mortality and morbidity [1]. In 2018 (World Health Organization 2019 report), nearly 228 million malaria cases occur worldwide, out of which 405,000 million die every year $[2,3]$. above $40 \%$ of the world's population in more than 80 countries and regions are still under the risky of contracting malaria.

About $80 \%$ of malaria death are concentrated in 15 countries most of them in Africa [4, 5]. IN recent, reduction in the number of malaria related cases are due to the global efforts of the current malaria interventions, such as decreasing mosquito breeding sites, sleeping under insecticide-treated nets (ITN), indoor residual spraying (IRS) with insecticides, are used for reducing malaria vectors and their bites, timely treatment with artemisinin-based combination therapies (ACTs) and chemoprevention for most vulnerable such as intermittent preventive treatment for pregnant women (IPTp) recommended by WHO. As global effort increases, it is necessary to know how these interventions can be implemented alongside one another.

Quarantine is also one of the public health control strategy of infectious diseases. The strategy focuses on isolation of infectious individuals from contacting with susceptible individuals or healthy populations. This control measure is effective to control and eliminate newly emerging infectious diseases caused by unidentified infectious agents.

Optimal control applications are important to approximate the efficacy of various policies and control measures. It is also important to cost estimation analysis of the examined control strategies. The theory of optimal control has been 
more successfully used in decision making in various applications after the development of Pontryagins's maximum principle (1962).

Mathematical models of the dynamics of malaria transmission are useful in providing a better insight into the behavior of the disease. These models have played a great role in influencing the decision making processes regarding intervention strategies for controlling and eliminating the spread of malaria. The study of malaria using mathematical modeling began in 1911 with Ronald Ross [6, 7]. Others have studied the transmission of malaria using SIR model for humans and SI for the mosquitoes. These are: Alemu Geleta Wedajo, Boka Kumsa Bole, Purnachandra Rao Koya [8, 9], Tuwiine, Mugisha and Luboobi [10] developed a compartment model for the spread of malaria with susceptible-infectedrecovered-susceptible (SIRS) pattern for human and susceptible-infected (SI) pattern for mosquitoes. Yang, Wei, and Li have proposed SIR for the human and SI for the vector compartment model and define the reproduction number, $R_{0}$ and show the existence and stability of the disease-free equilibrium and an endemic equilibrium [11].

Feng and Thieme formulated a perfect quarantine model where a proportion of infected people stay at home and do not infect anybody and showed that the model can give rise to sustained oscillations [12]. Hethcote et al. analyzed six types of SIQS and SIQR models to explore which one can produce periodic solutions [13]. Gumel et al used models to examine the effectiveness of quarantine and isolation on the control of SARS outbreaks [14]. Pandey et al developed a compartmental model for Ebola transmission to assess the effectiveness of non-pharmaceutical interventions for curtailing the epidemic in Liberia [15].

Erdem et al. studied the impact of imperfect quarantine on the dynamics of an SIR-type model [16]. X. Jin et al, mathematical analysis of the Ross-Macdonald malaria model with quarantine using SIQ-SI model type [17]. K. O. Okosun et al. (2013) derived and analyzed a malaria disease transmission mathematical model that includes insecticide treated net, treatment and indoor residual spray and applied optimal control strategy to study a possible treatment of infective humans that blocks transmission to mosquitoes in controlling the spread of malaria [18]. Suresh (1978) formulated and analyzed an optimal control problem with a simple epidemic model to examine effect of a quarantine program [19].

The purpose of the study of endemic malaria disease model system (1) with imperfect quarantine strategy is to reduce the number of susceptible mosquitoes bites from or contacts with malaria infectious humans and explore the effect of the strategy in the malaria control and elimination.

\section{Model Formulation}

The ordinary differential equations that describe the interactions between the human and mosquito population is formulated and described by Otieno et al. [20]. In this paper, a deterministic compartmental model is formulated and analyzed. The model is formulated based on the assumptions of [17] by incorporating imperfect quarantine that is, we classify the infectious human as Exposed quarantined individuals with no disease clinical symptoms for the time being, but sharing common environment or home with these may have continuous opportunities of bite from malaria parasite carrier denoted by $E_{q}$, Infected quarantined with disease clinical symptoms denoted by $I_{q}$ and Hospitalized (infected isolated individuals these are already getting treatment) and denoted by $H_{p}$.

The populations are subdivided into compartments according to the individual's disease status. We consider Eight-dimensional model. The human population as Susceptible $S_{h}$, Exposed quarantined $E_{q}$, Infected nonquarantined $I_{h}$, Infected quarantined $I_{q}$, Hospitalized (infected isolated) $H_{p}$, Recovered $R_{h}$. The mosquito populations as Susceptible $S_{v}$, and Infected $I_{v}$.

The total population sizes at time $t$, for humans are denoted and defied by $N_{h}(t)=S_{h}+E_{q}+I_{h}+I_{q}+H_{p}+$ $R_{h}$ and for mosquitoes are denoted and defied by $N_{v}(t)=$ $S_{v}+I_{v}$ respectively.

The susceptible humans $S_{h}$ are recruited at the rate, $\Lambda_{h}$. They either die from natural causes at a rate of $\mu_{h}$ or move to Susceptible quarantined human compartment class $S_{q}$ and Hospitalized human compartment class $H_{p}$ by acquiring malaria through contact with infectious mosquitoes with respective rates $\mu \lambda_{h}$ and $(1-\mu) \lambda_{h}$ respectively, Where $\mu$ and $(1-\mu)$ are the rates of susceptible humans joining susceptible quarantined and Hospitalized human compartments respectively, and $\lambda_{h}=\phi \omega \beta_{h} \frac{I_{v}}{N_{h}-\sigma\left(E_{q}+I_{q}+H\right)}$ is the force of infection from mosquito to human where, $\beta_{h}$ is the rate of probability of human getting infected, $\phi$ is the mosquito contact rate with human and $\omega$ is mosquito biting rate and $\sigma$ is the rate of reduction of mosquito bites for quarantined human compartments. Note that, $\sigma=1$ corresponds to perfect quarantine, $\sigma=0$ corresponds to no quarantine, and $0<\sigma<1$ corresponds to imperfect quarantine. The Infected non-quarantined individuals move to hospitalized (isolated class) with respective rate $\varphi$ or recovery class by getting partial immunity at a rate $\gamma$. They also die because of natural and disease induced death rates at $\mu_{h}$ and $\delta_{h}$ respectively. The Exposed quarantined individuals either die from natural causes at a rate of $\mu_{h}$ or move to Infected quarantined class $I_{q}$ after developing disease symptoms at a rate $\alpha_{h}$. Infected quarantined individuals $I_{q}$ are move to hospitalized (infected isolated) with respective rate $\varphi_{1}$ or recovery class by getting partial immunity at a rate $\gamma_{2}$. They also die because of natural and disease induced death rates at $\mu_{h}$ and $\delta_{h}$ respectively. Hospitalized (infected isolated human class) move to recovery class by getting partial immunity at a rate $\gamma_{1}$ or die because of natural and disease induced death rates because of natural and disease induced death rates at $\mu_{h}$ and $\delta_{h}$ respectively. These infectious individuals progress to partially immune group (recovered class), either partially immune group losses immunity and becomes again susceptible at a rate $\theta$ or die from natural death at a rate $\mu_{h}$. 
Susceptible mosquitoes $S_{v}$ are recruited at the rate $\Lambda_{v}$. They either die due to natural death at a rate of $\mu_{v}$ or move to Infected class $I_{v}$ by acquiring malaria through contact with infectious humans with respective rate $\lambda_{v}=\phi \omega \beta_{v} \frac{I_{h}+(1-\sigma)\left(E_{q}+I_{q}+H\right)}{N_{h}-\sigma\left(E_{q}+I_{q}+H\right)}$ where $\beta_{v}$ is the Probability of a mosquito getting infected. Infected mosquitoes $I_{v}$ are die because of natural and disease induced death rates $\mu_{v}$ and $\delta_{v}$ respectively.

From the law of conservation, the total number of bites by mosquitoes equal to the total number of bites on humans (i.e., $\phi \omega N_{v}=\phi \omega N_{h}$ implies $N_{v}=N_{h}$ ).

Table 1. State variables of the basic endemic malaria model.

\begin{tabular}{ll}
\hline Symbol & Description \\
\hline$S_{h}(t)$ & Number of Susceptible humans at time $t$ \\
$E_{q}(t)$ & Number of Exposed quarantined humans at time $t$ \\
$I_{h}(t)$ & Number of Infected non-quarantined humans at time $t$ \\
$I_{q}(t)$ & Number of infected quarantined humans at time $t$ \\
$H_{p}(t)$ & Number of hospitalized humans at time $t$ \\
$R_{h}(t)$ & Number of recovered humans at time $t$ \\
$S_{v}(t)$ & Number of Susceptible mosquitoes at time $t$ \\
$I_{v}(t)$ & Number of infectious mosquitoes at time $t$ \\
$N_{h}(t)$ & Total number of humans populations at time $t$ \\
$N_{v}(t)$ & Total number of mosquitoes populations at time $t$ \\
\hline
\end{tabular}

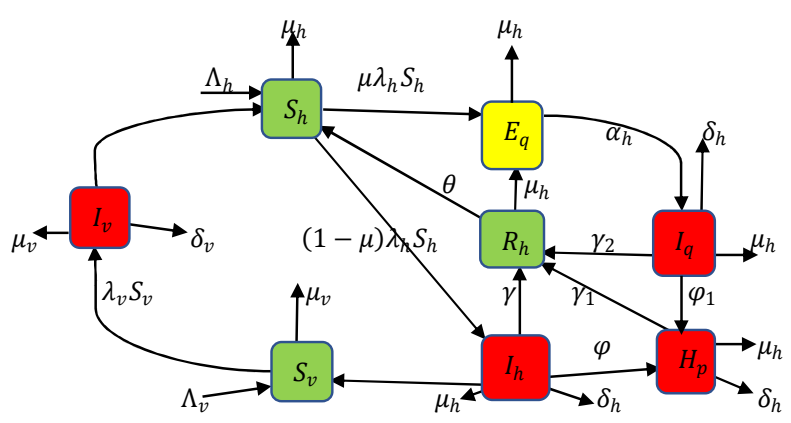

Figure 1. Dynamics of endemic malaria in humans and mosquito populations.

$$
\begin{gathered}
\frac{d S_{h}}{d t}=\Lambda_{h}+\theta R_{h}-\left(\lambda_{h}+\mu_{h}\right) S_{h} \\
\frac{d E_{q}}{d t}=\mu \lambda_{h} S_{h}-\left(\mu_{h}+\alpha_{h}\right) E_{q} \\
\frac{d I_{h}}{d t}=(1-\mu) \lambda_{h} S_{h}-\left(\mu_{h}+\delta_{h}+\varphi+\gamma\right) I_{h} \\
\frac{d I_{q}}{d t}=\alpha_{h} E_{q}-\left(\mu_{h}+\delta_{h}+\varphi_{1}+\gamma_{2}\right) I_{q} \\
\frac{d H_{p}}{d t}=\varphi I_{h}+\varphi_{1} I_{q}-\left(\mu_{h}+\delta_{h}+\gamma_{1}\right) H_{p} \\
\frac{d R_{h}}{d t}=\gamma I_{h}+\gamma_{2} I_{q}+\gamma_{1} H_{p}-\left(\theta+\mu_{h}\right) R_{h} \\
\frac{d S_{v}}{d t}=\Lambda_{v}-\left(\lambda_{v}+\mu_{v}\right) S_{v} \\
\frac{d I_{v}}{d t}=\lambda_{v} S_{v}-\left(\mu_{v}+\delta_{v}\right) I_{v}
\end{gathered}
$$

With initial conditions:

$$
\begin{gathered}
S_{h}(0)=S_{0 h} \geq 0, E_{q}(0)=E_{0 q} \geq 0, I_{h}(0)=I_{0 h} \geq 0, I_{q}(0)= \\
I_{0 q}, H_{p}(0)=H_{0 p}, R_{h}(0)=R_{0 h} \geq 0, S_{v}(0)=S_{0 v} \geq 0, I_{v}(0)= \\
I_{0 v} \geq 0, N_{h}(0)=N_{0 h} \geq 0, N_{v}(0)=N_{0 v} \geq 0 \\
\text { and } N_{h}(t)=S_{h}(t)+E_{q}(t)+I_{h}(t)+I_{q}(t)+H_{p}(t)+ \\
R_{h}(t) \text { and } N_{v}(t)=S_{v}(t)+I_{v}(t)
\end{gathered}
$$

The forces of infection on humans and mosquitoes respectively denoted and given by

$$
\lambda_{h}=\phi \omega \beta_{h} \frac{I_{v}}{N_{h}-\sigma\left(E_{q}+I_{q}+H_{p}\right)}, \lambda_{v}=\phi \omega \beta_{v} \frac{I_{h}+(1-\sigma)\left(E_{q}+I_{q}+H_{p}\right)}{N_{h}-\sigma\left(E_{q}+I_{q}+H_{p}\right)}
$$

\section{Model Analysis}

\subsection{Existence and Positivity of Solutions}

In this sub section, the malaria model governed by the system of equation (1) is epidemiologically and mathematically well posed will be shown. Its feasible region is also denoted and given by

$$
\Omega=\left\{\Omega_{\mathrm{h}} \times \Omega_{\mathrm{v}}\right\} \subset\left\{\mathbb{R}_{+}^{6} \times \mathbb{R}_{+}^{2}\right\}
$$
where,

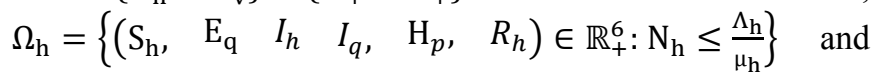
$\Omega_{\mathrm{v}}=\left\{\left(\mathrm{S}_{\mathrm{v}}, \mathrm{I}_{\mathrm{v}},\right) \in \mathbb{R}_{+}^{2}: \mathrm{N}_{\mathrm{v}} \leq \frac{\Lambda_{\mathrm{v}}}{\mu_{v}}\right\}$.

Theorem 1

The solution $\left\{S_{h}, E_{q}, \quad I_{h}, I_{q}, H_{p}, \quad R_{h}, S_{v}, I_{v}\right\}$ of the system of equation (1) $t$ is bounded and contained in the domain $\Omega$.

Proof : Let the solution of the system of equations (1) together with the positive initial conditions are $\Omega=$ $\left\{\begin{array}{llllllll}S_{h}, & E_{q} & I_{h} & I_{q}, & H_{p}, & R_{h}, & S_{v} & I_{v}\end{array}\right\}$ Also, let $N_{h}(t)=$ $S_{h}(t)+E_{q}(t)+I_{h}(t)+I_{q}(t)+H_{p}(t)+R_{h}(t)$ and $N_{v}(t)=$ $S_{v}(t)+I_{v}(t)$ The boundedness of both the human and mosquito populations are determined by the boundedness of $N_{h}(t)$ and $N_{v}(t)$ respectively.

Boundedness of $N_{h}(t)$ : Total sum of human compartments of the system of equation (1) leads to $\frac{d N_{h}}{d t}=\Lambda_{\mathrm{h}}$ $\mu_{h} N_{h}(t)-\delta_{h} I_{h}\left(t\right.$. After delating term $-\delta_{h} I_{h}(t)$, then without loss of generality we have $\frac{d N_{h}}{d t} \leq \Lambda_{\mathrm{h}}-\mu_{h} N_{h}(t)$ or equivalently $\frac{d N_{h}}{d t}+\mu_{h} N_{h}(t) \leq \Lambda_{\mathrm{h}}$ and its general solution is given by $N_{h}(t) \leq \frac{\Lambda_{\mathrm{h}}}{\mu_{\mathrm{h}}}+\left[N_{0 h}-\frac{\Lambda_{\mathrm{h}}}{\mu_{\mathrm{h}}}\right] \exp \left(-\mu_{\mathrm{h}} \mathrm{t}\right)$. As $\rightarrow \infty$, $N_{h}(t) \leq \frac{\Lambda_{\mathrm{h}}}{\mu_{\mathrm{h}}}$. Hence, the total human population is bounded i.e., $N_{0 h} \leq N_{h}(t) \leq \frac{\Lambda_{\mathrm{h}}}{\mu_{\mathrm{h}}}$.

Boundedness of $N_{v}(t)$ : total sum of mosquito compartments of the system of equations (1) leads to $\frac{d N_{v}}{d t}=$ $\Lambda_{v}-\mu_{v} N_{v}-\delta_{v} I_{v}$. After delating term $-\delta_{v} I_{v}$ then without loss of generality we have $\frac{d N_{v}}{d t} \leq \Lambda_{v}-\mu_{v} N_{v}(t)$, or equivalently $\frac{d N_{v}}{d t}+\mu_{v} N_{v}(t) \leq \Lambda_{v}$ and its general solution is given by $N_{v}(t) \leq \frac{\Lambda_{\mathrm{v}}}{\mu_{v}}+\left[N_{0 v}-\frac{\Lambda_{\mathrm{v}}}{\mu_{v}}\right.$. $] \exp \left(-\mu_{v} \mathrm{t}\right)$. As $t \rightarrow$ $\infty N_{v}(t) \leq \frac{\Lambda_{\mathrm{v}}}{\mu_{v}}$. Hence, the total mosquito population is 
bounded i.e. $N_{0 v} \leq N_{v}(t) \leq \frac{\Lambda_{\mathrm{v}}}{\mu_{v}}$.

Thus, the solutions of the model variables representing human populations $\left\{\left(\mathrm{S}_{\mathrm{h}}, \mathrm{E}_{\mathrm{q}} I_{h} \quad I_{q}, \mathrm{H}_{p} \quad R_{h}\right)\right\}$ are confined in the feasible region $\left.\Omega_{\mathrm{h}}=\left\{\begin{array}{llllll}\left(\mathrm{S}_{\mathrm{h}},\right. & \mathrm{E}_{\mathrm{q}} & I_{h} & I_{q}, & \mathrm{H}_{p} & R_{h}\end{array}\right) \in \mathbb{R}_{+}^{6}: \mathrm{N}_{\mathrm{h}} \leq \frac{\Lambda_{\mathrm{h}}}{\mu_{\mathrm{h}}}\right\}$

Similarly, the solutions of the model variables representing mosquito populations $\left\{\left(S_{v}, I_{v}\right)\right\}$ are confined in the feasible region $\Omega_{\mathrm{v}}=\left\{\left(\mathrm{S}_{\mathrm{v}}, \quad I_{v}\right) \in \mathbb{R}_{+}^{2}: \mathrm{N}_{\mathrm{v}} \leq \frac{\Lambda_{\mathrm{v}}}{\mu_{v}}\right\}$.

This shows that the feasible region of the model system (1) is bounded and is given by $\Omega=\left\{S_{h}(t), \quad E_{q}(t) \quad I_{h}(t), \quad I_{q}(t), \quad H_{p}(t), \quad R_{h}(t), \quad S_{v}(t), I_{v}(t)\right\} \in$ $\mathbb{R}_{+}^{8}$ or equivalently $\Omega=\left\{\Omega_{\mathrm{h}} \times \Omega_{\mathrm{v}}\right\} \subset\left\{\mathbb{R}_{+}^{6} \times \mathbb{R}_{+}^{2}\right\}$.

The Positivity of the model equations are stated and proved in the form of a theorem as follows:

Theorem 2: The solutions $\left\{S_{h}(t), \quad E_{q}(t) \quad I_{q}(t), \quad H_{p}(t), \quad R_{h}(t), \quad S_{v}(t), I_{v}(t)\right\} \quad$ of the malaria model system (1) together with the non-negative initial conditions are all non-negative for all $t>0$.

Proof:

Positivity of $S_{h}$ : Consider $\frac{d S_{h}}{d t}=\Lambda_{h}+\omega S_{q}+\theta R_{h}-$ $\left(\lambda_{h}+\mu_{h}\right) S_{h}$.

After delating terms $\Lambda_{h}$ and $\theta R_{h}$, then without loss of generality we have an inequality $\frac{d S_{h}}{d t} \geq-\left(\lambda_{h}+\mu_{h}\right) S_{h}$ or $\frac{d S_{h}}{d t} \geq-\left(\lambda_{h}+\mu_{h}\right) S_{h}$ and its general solution is given by

$S_{h}(t) \geq \exp \left[S_{0 h}-\left(\lambda_{h}+\mu_{h}\right) t\right] \geq 0$. Therefore $S_{h}(t) \geq$ 0 for $t>0$.

Positivity of $E_{q}$ : Consider $\frac{d E_{q}}{d t}=\mu \lambda_{h} S_{h}-\left(\mu_{h}+\alpha_{h}\right) E_{q}$. After delating the term $\mu \lambda_{h} S_{h}$, then without loss of generality we have an inequality $\frac{d E_{q}}{d t} \geq-\left(\mu_{h}+\alpha_{h}\right) E_{q}$ and its general solution is given by $E_{q}(t) \geq \exp \left[E_{0 q}-\left(\mu_{h}+\alpha_{h}\right) t\right] \geq 0$. Therefore $E_{q}(t) \geq 0$ for $t>0$.

Positivity of $I_{h}$ : Consider. $\frac{d I_{h}}{d t}=(1-\mu) \lambda_{h} S_{h}-$ $\left(\mu_{h}+\delta_{h}+\varphi+\gamma\right) I_{h}$. After delating the terms $(1-\mu) \lambda_{h} S_{h}$, then without loss of generality we have an inequality

$\frac{d I_{h}}{d t} \geq-\left(\mu_{h}+\delta_{h}+\varphi+\gamma\right) I_{h}$ and its general solution is given by $I_{h}(t) \geq \exp \left[I_{0 h}-\left(\mu_{h}+\delta_{h}+\varphi+\gamma\right) t\right] \geq 0$. Therefore $I_{h}(t) \geq 0$ for $t>0$.

Positivity of $I_{q}$ : Consider., $\frac{d I_{q}}{d t}=\alpha_{h} E_{Q}-\left(\mu_{h}+\delta_{h}+\varphi_{1}+\right.$ $\left.\gamma_{2}\right) I_{q}$. After delating the terms $\alpha_{h} E_{Q}$, then without loss of generality we have an inequality $\frac{d I_{h}}{d t} \geq-\left(\mu_{h}+\delta_{h}+\varphi_{1}+\right.$ $\left.\gamma_{2}\right) I_{q}$ and its general solution is given by $I_{q}(t) \geq \exp \left[I_{0 q}-\right.$ $\left.\left(\mu_{h}+\delta_{h}+\varphi_{1}+\gamma_{2}\right) t\right] \geq 0$. Therefore $I_{q}(t) \geq 0$ for $t>0$.

Positivity of $H_{p}$ : Consider $\frac{d H_{p}}{d t}=\varphi I_{h}+\varphi_{1} I_{q}$ $\left(\mu_{h}+\delta_{h}+\gamma_{1}\right) H_{p}$. After delating the terms $\varphi I_{h}$ and $\varphi_{1} I_{q}$, then without loss of generality we have an inequality $\frac{d H_{p}}{d t} \geq$ $-\left(\mu_{h}+\delta_{h}+\gamma_{1}\right) H_{p}$ and its general solution is given by: $\quad \mathrm{H}(t) \geq \exp \left[H_{0 \mathrm{p}}-\left(\mu_{h}+\delta_{h}+\gamma_{1}\right) t\right] \geq 0$.

Therefore $H_{p}(t) \geq 0$ for $t>0$.

Positivity of $R_{h}$ : Consider $\frac{d R_{h}}{d t}=\gamma I_{h}+\gamma_{2} I_{q}+\gamma_{1} H_{p}-$ $\left(\theta+\mu_{h}\right) R_{h}$. After delating terms $\gamma I_{h}, \gamma_{2} I_{q}$ and $\gamma_{1} H_{p}$, then without loss of generality we have an inequality $\frac{d R_{h}}{d t} \geq$ $-\left(\theta+\mu_{h}\right) R_{h}$ and its general solution is given by: $R_{h}(t) \geq$ $\exp \left[R_{0 h}-\left(\theta+\mu_{h}\right) t\right] \geq 0$. Therefore $R_{h}(t) \geq 0$ for $t>0$.

Positivity of $S_{v}$ : Consider $\frac{d S_{v}}{d t}=\Lambda_{v}-\left(\lambda_{v}+\mu_{v}\right) S_{v}$. After delating the term $\Lambda_{v}$, then without loss of generality we have an inequality $\frac{d S_{v}}{d t} \geq-\left(\lambda_{v}+\mu_{v}\right) S_{v}$ and its general solution is given by $S_{v}(t) \geq \exp \left[S_{0 v}-\left(\lambda_{v}+\mu_{v}\right) t\right] \geq 0$. Therefore $S_{v}(t) \geq 0$ for $t>0$.

Positivity of $I_{v}: \frac{d I_{v}}{d t}=\lambda_{v} S_{v}-\left(\mu_{v}+\delta_{v}\right) I_{v}$. After delating the term $\lambda_{v} S_{v}$, then without loss of generality we have an inequality $\frac{d I_{v}}{d t} \geq-\left(\mu_{v}+\delta_{v}\right) I_{v}$ and its general solution given by: $I_{v}(t) \geq \exp \left[I_{0 v}-\left(\mu_{v}+\delta_{v}\right) t\right] \geq 0$. Therefore $I_{v}(t) \geq$ 0 for $t>0$.

\subsection{Existence of Disease Free Equilibrium Points}

The disease-free equilibrium point of the model is its steady state solutions without infection or disease. Consider the disease free-equilibrium points denoted and given by:

$$
E_{0}=\left\{\begin{array}{llllllll}
S_{h}^{0}, & E_{q}^{0} & I_{h}^{0} & I_{q}^{0}, & H_{p}^{0}, & R_{h}^{0}, \quad S_{v}^{0}, \quad I_{v}^{0}
\end{array}\right\}
$$

where, $S_{h}^{0}, E_{h}^{0}, I_{h}^{0}, I_{q}^{0}, H_{p}^{0}, R_{h}^{0}, S_{v}^{0}$ and $I_{v}^{0}$ are the components of $E_{0}$ and $E_{h}^{0}=I_{h}^{0}=H_{p}^{0}=I_{q}^{0}=R_{h}^{0}=I_{v}^{0}=0$

and the non-infectious are obtained by setting $\frac{d S_{h}}{d t}=\frac{d S_{v}}{d t}=$ 0 in the malaria model system (1) and solving the resultant gives $S_{h}^{0}=\frac{\Lambda_{h}}{\mu_{h}}$ and similarly, gives $S_{v}^{0}=\frac{\Lambda_{v}}{\mu_{v}}$. Thus,

$$
E_{0}=\left\{\frac{\Lambda_{h}}{\mu_{h}}, \quad 0, \quad 0, \quad 0 \quad 0 \quad \frac{\Lambda_{v}}{\mu_{v}}, 0,0\right\}
$$

\subsection{Reproduction Number}

The basic reproduction number denoted by $R_{0}$ is the average number of secondary infectious infected by an infective individual during his or her whole course of disease in case that all of the population are susceptible [21]. It helps to explore whether an infection will expand through the population or go away from the population. In order to determine the stability of system (1) the threshold condition for the establishment of the disease is necessary to be obtained. Here the reproduction number is calculated using the next generation matrix method that is developed by van den Driessche and Watmough [22]. The local asymptotic stability occur if $R_{0}<1$ and instability occur if $R_{0}>1$. Now let the system (1) be rearranged by beginning with the infected classes as follows:

Let $X=\left(\mathrm{E}_{\mathrm{q}}, \quad \mathrm{I}_{\mathrm{h}}, \quad \mathrm{I}_{\mathrm{q}}, H_{p}, \quad \mathrm{I}_{\mathrm{v}}, \quad \mathrm{R}_{\mathrm{h}}, \quad S_{h}, S_{v}\right)^{T}$. Then the new infections be identified from all other class transitions in the population.

The infected classes among all the classes of both human host and mosquito vector are $\mathrm{E}_{\mathrm{q}}, \mathrm{I}_{\mathrm{h}}, \mathrm{I}_{\mathrm{q}}, \mathrm{H}_{p}$ and $\mathrm{I}_{\mathrm{v}}$. The vector of rates of the appearance of new infections in each compartment is denoted by $F$. Further, $V=V^{+}+V^{-}$where $V^{+}$is the vector rate of transfer into the particular compartment and $V^{-}$is the vector rate of transfer out of the 
particular compartment. In the model equations it is clear that there are four compartments for the infected. Thus,

$$
F\left(X_{i}\right)=\left[\begin{array}{c}
\mu \lambda_{h} S_{h} \\
(1-\mu) \lambda_{h} S_{h} \\
0 \\
0 \\
\lambda_{v} S_{v} \\
0 \\
0
\end{array}\right] \text { and } V\left(X_{i}\right)=\left[\begin{array}{c}
\left(\mu_{h}+\alpha_{h}\right) E_{q} \\
\left(\mu_{h}+\varphi+\delta_{h}+\gamma\right) I_{h} \\
-\alpha_{h} \alpha E_{q}+\left(\gamma_{2}+\mu_{h}+\varphi_{1}+\delta_{h}\right) I_{q} \\
-\varphi I_{h}-\varphi_{1} I_{q}+\left(\gamma_{1}+\mu_{h}+\delta_{h}\right) H_{p} \\
\left(\mu_{v}+\delta_{v}\right) I_{v} \\
0 \\
0
\end{array}\right]
$$

where,

$$
\begin{aligned}
& F=\frac{\partial F\left(X_{i}\right)}{\partial X_{i}}\left(E_{0}\right)=\left[\begin{array}{ccccc}
0 & 0 & 0 & 0 & \beta_{h} \omega \phi \mu \\
0 & 0 & 0 & 0 & (1-\mu) \beta_{h} \omega \phi \\
0 & 0 & 0 & 0 & 0 \\
0 & 0 & 0 & 0 & 0 \\
\frac{(1-\sigma) \beta_{v} \omega \phi S_{v}^{0}}{S_{h}^{0}} & \frac{\beta_{v} \omega \phi S_{v}^{0}}{S_{h}^{0}} & \frac{(1-\sigma) \beta_{v} \omega \phi S_{v}^{0}}{S_{h}^{0}} & \frac{(1-\sigma) \beta_{v} \omega \phi S_{v}^{0}}{S_{h}^{0}} & 0
\end{array}\right] \text { and } \\
& V=\frac{\partial V\left(X_{i}\right)}{\partial X_{i}}\left(E_{0}\right)=\left[\begin{array}{ccccc}
\left(\mu_{h}+\alpha_{h}\right) & 0 & 0 & 0 & 0 \\
0 & \left(\gamma+\mu_{h}+\varphi+\delta_{h}\right) & 0 & 0 & 0 \\
-\alpha_{h} & 0 & \left(\gamma_{2}+\mu_{h}+\varphi_{1}+\delta_{h}\right) & 0 & 0 \\
0 & -\varphi & -\varphi_{1} & \left(\gamma_{1}+\mu_{h}+\delta_{h}\right) & 0 \\
0 & 0 & 0 & 0 & \left(\mu_{v}+\delta_{v}\right)
\end{array}\right] \\
& F V^{-1}=\left[\begin{array}{ccccc}
0 & 0 & 0 & 0 & \frac{\beta_{h} \omega \phi \mu}{m_{5}} \\
0 & 0 & 0 & 0 & \frac{(1-u) \beta_{h} \omega \phi}{m_{5}} \\
0 & 0 & 0 & 0 & 0 \\
0 & 0 & 0 & 0 \\
\frac{(1-\sigma) \beta_{v} \omega \phi S_{v}^{0} \alpha_{h}\left(m_{4}+\varphi_{1}\right)}{m_{1} m_{3} m_{4} S_{h}^{0}} & \frac{\beta_{v} \omega \phi S_{v}^{0}\left(m_{4}+\varphi(1-\sigma)\right)}{m_{2} m_{4} S_{h}^{0}} & \frac{(1-\sigma) \beta_{v} \omega \phi S_{v}^{0}\left(m_{4}+\varphi_{1}\right)}{m_{3} m_{4} S_{h}^{0}} & \frac{(1-\sigma) \beta_{v} \omega \phi S_{v}^{0}}{m_{4} S_{h}^{0}} & 0 \\
\hline
\end{array}\right. \\
& \lambda=\sqrt{\frac{\beta_{h} \beta_{v} \omega^{2} \phi^{2} \mu_{h} \Lambda_{v}}{m_{1} m_{2} m_{3} m_{4} m_{4} \mu_{v} \Lambda_{h}}\left[m_{2}(1-\sigma)\left(m_{3} m_{4}+\alpha_{h}\left(m_{4}+\varphi_{1}\right)\right) \mu+m_{1} m_{3}\left(m_{4}+\varphi(1-\sigma)\right)(1-\mu)\right] .}
\end{aligned}
$$

Therefore; the basic reproduction number of the model system of (1) is denoted and given by

$$
R_{0}=\sqrt{\frac{\beta_{h} \beta_{v} \omega^{2} \phi^{2} \mu_{h} \Lambda_{v}}{m_{1} m_{2} m_{3} m_{4} m_{4} \mu_{v} \Lambda_{h}}\left[m_{2}(1-\sigma)\left(m_{3} m_{4}+\alpha_{h}\left(m_{4}+\varphi_{1}\right)\right) \mu+m_{1} m_{3}\left(m_{4}+\varphi(1-\sigma)\right)(1-\mu)\right]}
$$

Where, $m_{1}=\mu_{h}+\alpha_{h} m_{2}=\gamma+\mu_{h}+\varphi+\delta_{h}, m_{3}=\gamma_{2}+\mu_{h}+\varphi_{1}+\delta_{h}, m_{4}=\gamma_{1}+\mu_{h}+\delta_{h}$,

$$
m_{\mathbf{5}}=\mu_{v}+\delta_{v}
$$

\subsection{Global Stability of the Disease-Free Equilibrium Point}

To establish the global stability of disease free-equilibrium two conditions are considered. Castillo-Chavez et-al [23].

The model system (1) can be re-written in the following form

$$
\begin{gathered}
\frac{d X}{d t}=F(X, Z) \\
\frac{d Z}{d t}=G(X, Z), G(X, 0)=0
\end{gathered}
$$

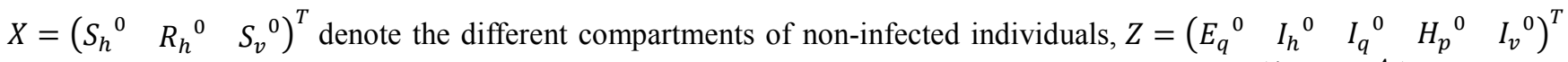
denote the different compartments of infectious individuals and $E_{0}=\left(X^{*}, Z^{*}\right)=\left(X^{*}, 0\right)$, where $X^{*}=\left(\begin{array}{lll}\Lambda_{h} & 0 & \frac{\Lambda_{v}}{\mu_{v}}\end{array}\right)$ denotes the disease free equilibrium of the model.

The point $\left(X^{*}, 0\right)$ is globally asymptotically stable for the model provided that $R_{0}<1$ and the following conditions hold.

(i) For $\frac{d X}{d t}=F(X, 0),\left(X^{*}, 0\right)$ is globally asymptotically stable 
(ii) $\mathrm{G}(X, Z)=A Z-\hat{G}(X, Z) \geq 0$ for all $(X, Z) \in \Omega$

Theorem 3 The disease free-equilibrium $E_{0}$ of model system (1) is globally asymptotically stable in $\Omega$ if $R_{0}<1$ an unstable if $R_{0}>1$.

Proof:

i) Solving the differential equation $\frac{d X}{d t}=F(X, 0)=\left\{\begin{array}{l}\Lambda_{h}-\mu_{h} S_{h}^{0} \\ -\left(\theta+\mu_{h}\right) \text { gives } \\ \Lambda_{v}-\mu_{v} S_{v}^{0}\end{array}\right.$

$S_{h}^{0}(t)=\frac{\Lambda_{h}}{\mu_{h}}-\frac{\Lambda_{h}}{\mu_{h}} e^{-t \mu_{h}}+S_{h}^{0}(0) e^{-t \mu_{h}}, S_{v}^{0}(t)=\frac{\Lambda_{v}}{\mu_{v}}-\frac{\Lambda_{v}}{\mu_{v}} e^{-t \mu_{h}}+S_{v}^{0}(0) e^{-t \mu_{h}}$ and $R_{h}^{0}(t)=R_{h}^{0}(0) e^{-t\left(\theta+\mu_{h}\right)}$.

As $t \rightarrow \infty, S_{h}^{0}(t) \rightarrow \frac{\Lambda_{h}}{\mu_{h}}, S_{v}^{0}(t) \rightarrow \frac{\Lambda_{v}}{\mu_{v}}$ and $R_{h}^{0}(t) \rightarrow 0$. Thus, $\left(X^{*}, 0\right)$ is globally and asymptotically stable.

ii) To show $\hat{G}(X, Z)=A Z-G(X, Z)$,

$$
\begin{gathered}
\text { Let } G(X, Z)=\left(\begin{array}{c}
-m_{1} E_{q} \\
-m_{2} I_{h} \\
\alpha_{h} E_{q}-m_{3} I_{q} \\
\varphi I_{h}+\varphi_{1} I_{q}-m_{4} H_{p} \\
-m_{5} I_{v}
\end{array}\right) \\
A=\frac{\partial G(X, Z)}{\partial Z}\left(X^{*}, 0\right)=\left(\begin{array}{ccccc}
-m_{1} & 0 & 0 & 0 & 0 \\
0 & -m_{2} & 0 & 0 & 0 \\
\alpha_{h} & 0 & -m_{3} & 0 & 0 \\
0 & \varphi & \varphi_{1} & -m_{4} & 0 \\
0 & 0 & 0 & 0 & -m_{5}
\end{array}\right)
\end{gathered}
$$

Which is Metzler-matrix whose non-negative off-diagonal elements.

$$
\begin{gathered}
A Z=\left(\begin{array}{ccccc}
-m_{1} & 0 & 0 & 0 & 0 \\
0 & -m_{2} & 0 & 0 & 0 \\
\alpha_{h} & 0 & -m_{3} & 0 & 0 \\
0 & \varphi & \varphi_{1} & -m_{4} & 0 \\
0 & 0 & 0 & 0 & -m_{5}
\end{array}\right)\left(\begin{array}{c}
E_{q} \\
I_{h} \\
I_{q} \\
H_{p} \\
I_{v}
\end{array}\right) \\
\widehat{G}(X, Z)=\left(\begin{array}{c}
-m_{1}\left(E_{q}-E_{q}\right) \\
-m_{2}\left(I_{h}-I_{h}\right) \\
\varphi\left(I_{h}-I_{h}\right)+\varphi_{1}\left(I_{q}-I_{q}\right)-m_{4}\left(H_{p}-H_{p}\right) \\
-m_{5}\left(I_{v}-I_{v}\right)
\end{array}\right) \geq 0
\end{gathered}
$$

That is, $\widehat{G}(X, Z)=\left(\begin{array}{lllll}0 & 0 & 0 & 0 & 0\end{array}\right)^{T}$. Thus, $\widehat{G}(X, Z)=0$.

\subsection{Existence of Endemic Equilibrium Points}

Let the endemic equilibrium point be denoted by $E^{* *}=\left\{S_{h}^{* *}, S_{q}^{* *} I_{q}^{* *}, H^{* *}, R_{h}^{* *}, \quad S_{v}^{* *}, \quad I_{v}^{* *}\right\}$. It is the non-trivial positive equilibrium of the malaria model system (1). Each component of $E_{* *}$ is obtained by setting the right hand sides of all model system (1) equal to zero i.e.

$$
\begin{gathered}
\Lambda_{h}+\theta R_{h}^{* *}-\left(\lambda_{h}^{* *}+\mu_{h}\right) S_{h}^{* *}=0 \\
\left(1-u_{1}\right) \mu \lambda_{h}^{* *} S_{h}^{* *}-\left(\mu_{h}+\alpha_{h}\right) E_{q}{ }^{* *}=0 \\
(1-\mu) \lambda_{h}^{* *} S_{h}^{* *}+\tau \varphi I_{q}^{* *}-\left(\mu_{h}+\delta_{h}+\gamma_{1}\right) I_{h}{ }^{* *}=0 \\
\alpha_{h} E_{q}^{* *}-\left(\mu_{h}+\delta_{h}+\varphi_{1}+\gamma_{2}\right) I_{q}{ }^{* *}=0 \\
\varphi I_{h}^{* *}+\varphi_{1} I_{q}^{* *}-\left(\mu_{h}+\delta_{h}+\gamma_{1}\right) H_{p}{ }^{* *}=0 \\
\gamma I_{h}{ }^{* *}+\gamma_{2} I_{q}^{* *}+\gamma_{1} H^{* *}-\left(\theta+\mu_{h}\right) R_{h}^{* *}=0 \\
\Lambda_{v}-\left(\lambda_{v}^{* *}+\mu_{v}\right) S_{v}^{* *}=0 \\
\lambda_{v}^{* *} S_{v}^{* *}-\left(\mu_{v}+\delta_{v}\right) I_{v}^{* *}=0
\end{gathered}
$$

Up on computing the resultant equations as listed above, the components of $E^{*}$ are obtained as follows:

$$
S_{h}^{* *}=\frac{\Lambda_{h} m m_{1} m_{2} m_{3} m_{4}}{\left(\lambda_{h}^{* *}+\mu_{h}\right) m m_{1} m_{2} m_{3} m_{4}-\theta\left[m_{2} \alpha_{h}\left(\varphi_{1} \gamma_{1}+m_{4} \gamma_{2}\right) \mu+m_{1} m_{3}\left(\varphi \gamma_{1}+m_{4} \gamma\right)(1-\mu)\right] \lambda_{h}^{* *}}
$$




$$
\begin{aligned}
& E_{q}^{* *}=\frac{\mu \lambda_{h}^{* *} S_{h}^{* *}}{\mathrm{~m}_{1}} \\
& I_{h}^{* *}=\frac{(1-\mu) \lambda_{h}^{* *} S_{h}^{* *}}{m_{2}} \\
& I_{q}^{* *}=\frac{\alpha_{h} \mu \lambda_{h}^{* *} S_{h}^{* *}}{m_{1} m_{3}} \\
& H_{p}^{* *}=\frac{\left[m_{2} \varphi_{1} \alpha_{h} \mu+m_{1} m_{3} \varphi(1-\mu)\right] \lambda_{h}^{* *} S_{h}^{* *}}{m_{1} m_{2} m_{3} m_{4}} \\
& R_{h}^{* *}=\frac{\left[m_{2} \alpha_{h}\left(\varphi_{1} \gamma_{1}+m_{4} \gamma_{2}\right) \mu+m_{1} m_{3}\left(\varphi \gamma_{1}+m_{4} \gamma\right)(1-u)\right] \lambda_{h}^{* *} s_{h}^{* *}}{m m_{1} m_{2} m_{3} m_{4}} \\
& S_{v}^{* *}=\frac{\Lambda_{v}}{\lambda_{v}^{+}+\mu_{v}} \\
& I_{v}^{* *}=\frac{\lambda_{v}^{* *} \Lambda_{v}}{m_{5}\left(\lambda_{v}^{* *}+\mu_{v}\right)} \\
& \lambda_{h}^{* *}=\beta_{h} \omega \phi \frac{I_{v}^{* *}}{N_{h}^{* *}-\sigma\left(E_{q}^{* *}+I_{q}^{* *}+H_{P}^{* *}\right)} \\
& \lambda_{v}^{* *}=\beta_{v} \omega \phi \frac{I_{h}^{* *}+(1-\sigma)\left(E_{q}^{* *}+I_{q}^{* *}+H_{P}^{* *}\right)}{N_{h}^{* *}-\sigma\left(E_{q}^{* *}+I_{q}^{* *}+H_{P}^{* *}\right)}
\end{aligned}
$$

Where, $N_{h}^{* *}=S_{h}^{* *}+E_{q}^{* *}+I_{h}^{* *}+I_{q}^{* *}+H_{P}^{* *}+R_{h}^{* *}$

After substation of (5) in to (6) and (7), the re-arranged and simplified of (6) and (7) in terms of $\lambda_{h}^{* *}$ gives the following quadratic equation

$$
a\left(\lambda_{h}^{* *}\right)^{2}+b \lambda_{h}^{* *}+c=0
$$

Where,

$$
\begin{gathered}
a=\Lambda_{h} m_{5}(K+L)\left(\mu_{v}(K+L)+\beta_{v} \phi \omega L\right) \\
b=\Lambda_{h} m m_{1} m_{2} m_{3} m_{4}\left[2 \mu_{v}(K+L)+\beta_{v} \phi \omega L\right]-\phi^{2} \omega^{2} \beta_{h} \beta_{v} \Lambda_{v} L\left(m m_{1} m_{2} m_{3} m_{4}-\theta K\right) \\
c=m_{1} m_{2} m_{3} m_{4} m_{5} \mu_{v} \Lambda_{h}\left(1-R_{0}^{2}\right) \\
K=m_{2} \alpha_{h}\left(\varphi_{1} \gamma_{1}+m_{4} \gamma_{2}\right) \mu+m_{1} m_{3}\left(\varphi \gamma_{1}+m_{4} \gamma\right)(1-u) \\
L=m\left[m_{2}(1-\sigma)\left(m_{3} m_{4}+\alpha_{h}\left(m_{4}+\varphi_{1}\right)\right) \mu+m_{1} m_{3}\left(m_{4}+\varphi(1-\sigma)\right)(1-\mu)\right]
\end{gathered}
$$

In (8) $\lambda_{h}^{* *}=0$ corresponds to the disease free-equilibrium $E_{0}$. If $\lambda_{h}^{* *} \neq 0$, then existence of endemic equilibria is computed by quadratic equation $a\left(\lambda_{h}^{* *}\right)^{2}+b \lambda_{h}^{* *}+c=0$. Note that that the coefficient $a>0, c>0$ if $R_{0}<1 c<0$ if $R_{0}>0$. The coefficient $b$ may expressed as

$$
b=\frac{m^{2} m^{2}{ }_{1} m_{2}^{2}{ }_{2} m_{3}{ }_{3} m_{4}{ }^{2} m_{5} \mu_{v} \Lambda_{h}}{\mu_{h}}\left(R_{c}^{2}-R_{0}^{2}\right)
$$

$$
\text { Where, } \begin{aligned}
R_{c} & =\sqrt{\frac{\mu_{h}(2 Y+Z)}{m^{2} m^{2}{ }_{1} m^{2}{ }_{2} m^{2}{ }_{3} m_{4}{ }^{2} m_{5} \mu_{v} \Lambda_{h}}}, \\
Y & =m m_{1} m_{2} m_{3} m_{4} m_{5} \mu_{v} \Lambda_{h}(K+L),
\end{aligned}
$$

$Z=\beta_{v} \phi \omega L\left(m m_{1} m_{2} m_{3} m_{4} m_{5} \mu_{v} \Lambda_{h}+\beta_{h} \phi \omega \theta K\right)$

Thus, the number of endemic equilibria of model (1) depends on the coefficients $a, b$ and $c$ as follows:

Theorem 4 The model system (1) has:

(i) A unique endemic equilibrium if $c<0$ that is, $R_{0}>1$.

(ii) A unique endemic equilibrium if $b<0$ and $c=0$ or $b<0, c>0$ and $\Delta=b^{2}-4 a c=0$ (iii) Two endemic equilibria where if $b\langle 0, c\rangle 0$ and $\Delta=b^{2}-4 a c>0$.

(iv) there are no endemic equilibria otherwise

From epidemiological perspective, condition (iii) of theorem 4 above implies that, $R_{0}<1$ has no longer guarantee for the elimination of the disease in the population. A new small threshold or saddle-node threshold for $R_{0}$ must be determined. To this aim we now express condition (iii) of theorem 4 in terms of basic reproduction number $R_{0}$ as follows. Not that, coefficient $b<0$ is equivalent to $R_{0}>$ $R_{c}$ and $c>0$ is equivalent to $R_{0}<1$. And also $b^{2}-4 a c>$ 0 is equivalent to

$$
a_{0} R_{0}{ }^{4}+b_{0} R_{0}{ }^{2}+c_{0}=0
$$

Where,

$$
a_{0}=\frac{m^{4} m_{1}^{4} m_{2}{ }_{2} m^{4}{ }_{3} m_{4}{ }^{4} m_{5}^{2} \mu_{v}^{2} \Lambda_{h}^{2}}{\mu_{h}{ }^{2}}>0
$$




$$
\begin{aligned}
& b_{0}=\frac{m^{2} m^{2}{ }_{1} m^{2}{ }_{2} m^{2}{ }_{3} m_{4}{ }^{2} m_{5} \mu_{v} \Lambda_{h}\left(2\left(a \mu_{h}-Y\right)-Z\right)}{\mu_{h}} \\
& c_{0}=Z^{2} \text { where, } Z=\beta_{v} \phi \omega L\left(m m_{1} m_{2} m_{3} m_{4} m_{5} \mu_{v} \Lambda_{h}+\beta_{h} \phi \omega \theta K\right)>0
\end{aligned}
$$

Equation (9) admits positive real roots if and only if $b_{0}<0$ and $\Delta_{0}=b_{0}^{2}-4 a_{0} c_{0} \geq 0$.

We can write

$$
\Delta_{1}=\frac{16 m^{4} m^{4}{ }_{1} m^{4}{ }_{2} m^{4}{ }_{3} m_{4}{ }^{4} m_{5}^{2} \mu_{\nu}^{2} \Lambda_{h}^{2} \pi\left[a_{0} \mu_{h}-(Y+Z)\right]}{\mu_{h}{ }^{2}} \text { Where, } \pi=a \mu_{h}-Y
$$

Choosing $\pi<0$ ensures that $a \mu_{h}-(Y+Z)<0$ and setting

$$
R_{ \pm}=\sqrt{\frac{-b_{0} \pm \sqrt{\Delta_{1}}}{2 a_{0}}}=\sqrt{\frac{\mu_{h}}{m^{2} m^{2}{ }_{1} m^{2}{ }_{2} m^{2}{ }_{3} m_{4}{ }^{2} m_{5} \mu_{v} \Lambda_{h}}}(\sqrt{-(\pi-Z)(1 \pm \sqrt{-\pi})})
$$

It follows that condition (iii) of theorem 4 is equivalent to $R_{c}<R_{0}<\min \left(1, R_{-}\right)$or $\max \left(1, R_{+}\right)<R_{c}<R_{0}<1$.

\section{Bifurcation Analysis}

The sub-threshold occurrence of multiple endemic equi libria stated in Theorem 4, is the result of forward or back ward at $R_{0}=1$. Now, we study the Centre manifold near the criticality by using the approach developed in [24, 25, 26]. Based on Center Manifold theory (Gumel and Song, 2008; Castillo-Chavez and Song, 2004) and general Centre manifold theory [27], we carry out a bifurcation analysis of model system (1) at $R_{0}=1$. Not that, the normal form representing the dynamics of the system on the Centre manifold is given by $\dot{y}=a y^{2}+b \xi y$, where,

$$
\begin{aligned}
& a=\frac{v}{2} . D_{x x} f\left(x_{0}, 0\right) w^{2}=\frac{1}{2} \sum_{k, i, j=1}^{n} v_{k} w_{i} w_{j} \frac{\partial^{2} f_{k}}{\partial x_{i} \partial x_{j}}\left(x_{0}, 0\right) \neq 0 \text { for } \mathrm{j}=1,2 \ldots, \mathrm{n} \\
& b=V \cdot D_{x \xi} f\left(x_{0}, 0\right) w=\sum_{k, i=1}^{n} v_{k} w_{i} \frac{\partial^{2} f_{k}}{\partial x_{i} \partial \xi}\left(x_{0}, 0\right) \neq 0 \text { for } \mathrm{i}=1,2 \ldots, \mathrm{n}
\end{aligned}
$$

Here, the symbol $\xi$ denotes a bifurcation parameter to be chosen, $f_{k} \mathrm{~s}$ denote the right hand side of system (1), $x$ denotes the state vector, $x_{0}$ the disease-free equilibrium $E_{0}$, $D_{x}$ denotes the differential operator with respect to $x, D_{\xi}$ denotes the differential operator with respect to $\xi$, and $w$ and $v$ denote the right and left eigenvectors, respectively, corresponding to the null eigenvalue of the Jacobian matrix of system (1), evaluated at $x_{0}$ for $\xi=0$.

To apply the above result, the following simplification and change of variables are made on system (1). Let

$$
S_{h}=x_{1}, E_{q}=x_{2}, I_{h}=x_{3}, I_{q}=x_{4}, H_{p}=x_{5}, R_{h}=x_{6},
$$
$S_{v}=x_{7}$, and $I_{v}=x_{8}$, so, $N_{h}=x_{1}+x_{2}+x_{3}+x_{4}+x_{5}+$ $x_{6}$ and $N_{v}=x_{7}+x_{8}$. Mor over, by using the vector

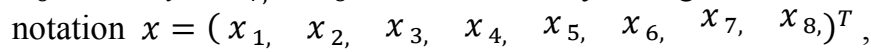
the system (1) can be written in the form $\frac{d x}{d t}=\left(f_{1}, f_{2}, f_{3}, f_{4}, f_{5}, f_{6}, f_{7}, f_{8},\right)^{T}$ as follows

$$
\begin{gathered}
\frac{d x_{1}}{d t}=f_{1}=\Lambda_{h}+\theta R_{h}-\left(\beta_{h} \omega \phi \frac{x_{8}}{x_{1}+x_{2}+x_{3}+x_{4}+x_{5}+x_{6}-\sigma\left(x_{2}+x_{4}+x_{5}\right)}+\mu_{h}\right) x_{1} \\
\frac{d x_{2}}{d t}=f_{2}=\mu \beta_{h} \omega \phi \frac{x_{8}}{x_{1}+x_{2}+x_{3}+x_{4}+x_{5}+x_{6}-\sigma\left(x_{2}+x_{4}+x_{5}\right)} x_{1}-\left(\mu_{h}+\alpha_{h}\right) x_{2} \\
\frac{d x_{3}}{d t}=f_{3}=(1-\mu) \beta_{h} \omega \phi \frac{x_{8}}{x_{1}+x_{2}+x_{3}+x_{4}+x_{5}+x_{6}-\sigma\left(x_{2}+x_{4}+x_{5}\right)} x_{1}-\left(\mu_{h}+\delta_{h}+\varphi+\gamma\right) x_{3} \\
\frac{\frac{d x_{4}}{d t}=f_{4}=\alpha_{h} x_{2}-\left(\mu_{h}+\delta_{h}+\varphi_{1}+\gamma_{2}\right) I_{q}}{d t}=f_{5}=\varphi x_{3}+\varphi_{1} x_{4}-\left(\mu_{h}+\delta_{h}+\gamma_{1}\right) x_{5} \\
\frac{d x_{6}}{d t}=f_{6}=\gamma x_{3}+\gamma_{2} x_{4}+\gamma_{1} x_{5}-\left(\theta+\mu_{h}\right) x_{6} \\
\frac{d x_{7}}{d t}=f_{7}=\Lambda_{v}-\left(\beta_{v} \omega \phi \frac{x_{3}+(1-\sigma)\left(x_{2}+x_{4}+x_{5}\right)}{x_{1}+x_{2}+x_{3}+x_{4}+x_{5}+x_{6}-\sigma\left(x_{2}+x_{4}+x_{5}\right)}+\mu_{v}\right) x_{7}
\end{gathered}
$$




$$
\frac{d x_{8}}{d t}=f_{8}=\beta_{v} \omega \phi \frac{x_{3}+(1-\sigma)\left(x_{2}+x_{4}+x_{5}\right)}{x_{1}+x_{2}+x_{3}+x_{4}+x_{5}+x_{6}-\sigma\left(x_{2}+x_{4}+x_{5}\right)} x_{7}-\left(\mu_{v}+\delta_{v}\right) x_{8}
$$

We choose the rate of transmission of infection from an infectious mosquito to a susceptible human, $\beta_{h}$, as the bifurcation parameter. We observe that $R_{0}=1$ is equivalent to:

$$
\beta_{h}=\beta_{h}^{*}=\frac{m_{1} m_{2} m_{3} m_{4} m_{5} \mu_{v} \Lambda_{h}}{\beta_{v} \phi^{2} \omega^{2}\left[m_{2}(1-\sigma)\left(m_{3} m_{4}+\alpha_{h}\left(m_{4}+\varphi_{1}\right)\right) \mu+m_{1} m_{3}\left(m_{4}+\varphi(1-\sigma)\right)(1-\mu)\right]}
$$

So that the disease free-equilibrium $E_{0}$ is locally asymptotically stable when $\beta_{h}<\beta_{h}^{*}$ and unstable when $\beta_{h}>\beta_{h}^{*}$. Hence, $\beta_{h}=\beta_{h}^{*}$ is a bifurcation value.

The Jacobian matrix of system (1) evaluated at $E_{0}$ for $\beta_{h}=\beta_{h}^{*}$ is given by

$$
J\left(E_{0}, \beta_{h}^{*}\right)=\left(\begin{array}{cccccccc}
-\mu_{h} & 0 & 0 & 0 & 0 & \theta & 0 & -J_{18} \\
0 & -m_{1} & 0 & 0 & 0 & 0 & 0 & J_{28} \\
0 & 0 & -m_{2} & 0 & 0 & \theta & 0 & J_{38} \\
0 & \alpha_{h} & 0 & -m_{3} & 0 & 0 & 0 & 0 \\
0 & 0 & \varphi & \varphi_{1} & -m_{4} & 0 & 0 & 0 \\
0 & 0 & \gamma & \gamma_{2} & \gamma_{1} & -m & 0 & 0 \\
0 & -J_{72} & -J_{73} & -J_{74} & -J_{75} & 0 & -\mu_{v} & 0 \\
0 & J_{82} & J_{83} & J_{84} & J_{85} & 0 & 0 & -m_{5}
\end{array}\right)
$$

Where,

$$
\begin{gathered}
J_{18}=\omega \phi \beta_{h}^{*}, J_{28}=\mu \omega \phi \beta_{h}^{*}, J_{38}=(1-\mu) \omega \phi \beta_{h}^{*}, J_{73}=J_{83}=\frac{\omega \phi \beta_{v} S_{v}^{0}}{S_{h}^{0}} \\
J_{72}=J_{74}=J_{75}=J_{82}=J_{84}=J_{85}=\frac{(1-\sigma) \omega \phi \beta_{v} S_{v}^{0}}{S_{h}^{0}}
\end{gathered}
$$

$\operatorname{det}\left(J\left(E_{0}\right)-\lambda I_{6}\right)=0$, Since the first and seventh columns contain only diagonal terms they give two negative eigenvalues i.e., $\lambda_{1}=-\mu_{h}, \lambda_{2}=-\mu_{v}$, then deleting rows and columns of the first and fifth of $J\left(E_{0}\right)$ we have:

$$
J_{1}\left(E_{0}, \beta_{h}^{*}\right)=\left(\begin{array}{cccccc}
-m_{1} & 0 & 0 & 0 & 0 & J_{28} \\
0 & -m_{2} & 0 & 0 & 0 & J_{38} \\
\alpha_{h} & 0 & m_{3} & 0 & 0 & 0 \\
0 & \varphi & \varphi_{1} & -m_{4} & 0 & 0 \\
0 & \gamma & \gamma_{2} & \gamma_{1} & -m & 0 \\
J_{82} & J_{83} & J_{84} & J_{85} & 0 & -m_{5}
\end{array}\right)
$$

In the same way, the fifth column of $J_{1}\left(E_{0}\right)$ contains only diagonal term which also forms a negative eigenvalue i.e., $\lambda_{3}=-m$. The remaining five eigenvalues are obtained from the sub-matrix

$$
J_{2}\left(E_{0}, \beta_{h}^{*}\right)=\left(\begin{array}{ccccc}
-m_{1} & 0 & 0 & 0 & J_{28} \\
0 & m_{2} & 0 & 0 & J_{38} \\
\alpha_{h} & 0 & -m_{3} & 0 & 0 \\
0 & \varphi & \varphi_{1} & -m_{4} & 0 \\
J_{83} & J_{84} & J_{85} & 0 & -m_{5}
\end{array}\right)
$$

The eigen values of the matrix $J_{2}\left(E_{0}\right)$ are the roots of the characteristic equation

$$
\lambda^{5}+A_{1} \lambda^{4}+A_{3} \lambda^{3}+A_{2} \lambda^{2}+A_{1} \lambda+A_{5}=0
$$

Where,

$$
\begin{gathered}
A_{1}=m_{1}+m_{2}+m_{3}+m_{4}+m_{5} \\
A_{2}=m_{1} m_{2}+m_{3} m_{4}+\left(m_{1}+m_{2}\right)\left(m_{3}+m_{4}\right)+m_{5}\left(m_{1}+m_{2}+m_{3}+m_{4}\right)-\frac{\beta_{h} \beta_{v} \omega^{2} \phi^{2} \mu_{h} \Lambda_{v}}{\mu_{v} \Lambda_{h}}[(1-\sigma) \mu+(1-\mu)] \\
-\frac{A_{3}=m_{1} m_{2}+m_{3} m_{4}+\left(m_{1}+m_{2}\right)\left(m_{3}+m_{4}\right)+m_{5}\left(m_{1}+m_{2}+m_{3}+m_{4}\right)}{\mu_{v} \Lambda_{h}}\left[\left(m_{2}+m_{3}+m_{4}+\alpha_{h}\right) \mu+\left(\varphi(1-\sigma)+m_{1}+m_{3}+m_{4}\right)(1-\mu)\right]
\end{gathered}
$$




$$
\begin{gathered}
A_{4}=m_{1}+m_{2}+m_{3}+m_{4}+m_{5}\left[m_{3} m_{4}\left(m_{1}+m_{2}\right)+m_{1} m_{2}\left(m_{3}+m_{4}\right)\right] \\
-\frac{\beta_{h} \beta_{v} \omega^{2} \phi^{2} \mu_{h} \Lambda_{v}}{\mu_{v} \Lambda_{h}}\left[\left[\left(m_{2} m_{3}+m_{4}\left(m_{2}+m_{3}\right)+\alpha_{h}\left(\varphi_{1}+m_{2}+m_{4}\right)\right)\right] \mu\right. \\
\left.+\left(\left(\varphi(1-\sigma)+m_{4}\right)\left(m_{1}+m_{3}\right)+m_{1} m_{3}\right)(1-\mu)\right] \\
A_{5}=\left(1-R_{0}^{2}\right) m_{1} m_{2} m_{3} m_{4} m_{5} \\
\lambda\left(\lambda^{4}+\mathrm{A}_{1} \lambda^{3}+\mathrm{A}_{3} \lambda^{2}+\mathrm{A}_{2} \lambda+\mathrm{A}_{1}\right)=0
\end{gathered}
$$

Thus, (16) implies that the Jacobian $J\left(E_{0}, \beta_{h}^{*}\right)$ of the compute the coefficients (10) and (11), we determine the linearized system has a simple zero eigenvalue and the other eigenvalues have negative real part. Therefore the diseasefree equilibrium $E_{0}$ is a nonhyperbolic equilibrium. To right and left eigenvectors corresponding to the zero eigenvalue. The components $w_{i}$, for $i=1, \ldots, 8$, of the right eigenvectors $w_{i}^{\prime} s$ are given by

$$
\begin{gathered}
-\mu_{h} w_{1}+\theta w_{6}-\omega \phi \beta_{h}^{*} w_{8}=0 \\
-m_{1} w_{2}+\omega \mu \phi \beta_{h}^{*} w_{8}=0 \\
-m_{2} w_{3}+\omega(1-\mu) \phi \beta_{h}^{*} w_{8}=0 \\
\alpha_{h} w_{2}-m_{3} w_{4}=0 \\
\varphi w_{3}+\varphi_{1} w_{4}-m_{4} w_{5}=0 \\
\gamma w_{3}+\gamma_{2} w_{4}+\gamma_{1} w_{5}-m w_{6}=0 \\
-\omega \phi \beta_{v} \frac{S_{v}^{0}}{S_{h}^{0}} w_{3}-(1-\sigma) \omega \phi \beta_{v} \frac{S_{v}^{0}}{S_{h}^{0}}\left(w_{2}+w_{4}+w_{5}\right)-\mu_{v} w_{7}=0 \\
\omega \phi \beta_{v} \frac{S_{v}^{0}}{S_{h}^{0}} w_{3}+(1-\sigma) \omega \phi \beta_{v} \frac{S_{v}^{0}}{S_{h}^{0}}\left(w_{2}+w_{4}+w_{5}\right)-m_{5} w_{8}=0
\end{gathered}
$$

Analogs the components of $v_{i}$, for $i=1, \ldots, 8$ of the left eigenvector are $v$ is given by

$$
\begin{gathered}
-\mu_{h} v_{1}=0 \\
m_{1} v_{2}+\alpha_{h} v_{4}-(1-\sigma) \omega \phi \beta_{v} \frac{S_{v}^{0}}{S_{h}^{0}}\left(w_{7}-w_{8}\right)=0 \\
-m_{2} v_{3}+\varphi v_{5}+\gamma v_{6}-\omega \phi \beta_{v} \frac{S_{v}^{0}}{S_{h}^{0}}\left(w_{7}-w_{8}\right)=0 \\
-m_{3} v_{4}+\varphi_{1} v_{5}+\gamma_{1} v_{6}-(1-\sigma) \omega \phi \beta_{v} \frac{S_{v}^{0}}{S_{h}^{0}}\left(w_{7}-w_{8}\right)=0 \\
-m_{4} v_{5}+\gamma_{2} v_{6}-(1-\sigma) \omega \phi \beta_{v} \frac{S_{v}^{0}}{S_{h}^{0}}\left(w_{7}-w_{8}\right)=0 \\
\theta v_{1}-k_{3} v_{4}-m v_{6}=0 \\
-\mu_{v} v_{7}=0 \\
-\omega \phi \beta_{h}^{*} v_{1}+\omega \mu \phi \beta_{h}^{*} v_{2}+\omega(1-\mu) \phi \beta_{h}^{*} v_{3}-m_{5} v_{8}=0
\end{gathered}
$$

There fore; for $w_{8}>0, v_{8}>0$ we have,

$$
\begin{gathered}
w_{1}=\omega \phi \beta_{h}^{*}\left[\frac{\left[m_{2} \alpha_{h}\left(\varphi_{1} \gamma_{1}+m_{4} \gamma_{2}\right) \mu+m_{1} m_{3}\left(\varphi \gamma_{1}+m_{4} \gamma\right)(1-u)\right]-m m_{1} m_{2} m_{3} m_{4}}{\mu_{h} m m_{1} m_{2} m_{3} m_{4}}\right] w_{8}, \\
w_{2}=\frac{\omega \mu \phi \beta_{h}^{*}}{m_{1}} w_{8}, w_{3}=\frac{(1-\mu) \omega \phi \beta_{h}^{*}}{m_{2}} w_{8} w_{4}=\frac{\alpha_{h} \omega \mu \phi \beta_{h}^{*}}{m_{1} m_{3}} w_{8}, w_{5}=\frac{\omega \phi \beta_{h}^{*}\left[m_{2} \alpha_{h} \mu+m_{1} m_{3} \varphi(1-\mu)\right]}{m_{1} m_{2} m_{3} m_{4}} w_{8} \\
, w_{6}=\omega \phi \beta_{h}^{*} \frac{\left[m_{2} \alpha_{h}\left(\varphi_{1} \gamma_{1}+m_{4} \gamma_{2}\right) \mu+m_{1} m_{3}\left(\varphi \gamma_{1}+m_{4} \gamma\right)(1-u)\right]}{m m_{1} m_{2} m_{3} m_{4}} w_{8}, w_{7}=-\frac{m_{5}}{\mu_{v}} \text { and }
\end{gathered}
$$




$$
\begin{gathered}
v_{1}=v_{6}=v_{7}=0, v_{2}=\frac{(1-\sigma) \omega \phi \beta_{v} S_{v}^{0}}{S_{h}^{0}}\left(\frac{m_{2} \alpha_{h}\left(\varphi_{1}+m_{4}\right)+m_{3} m_{4}}{m_{1} m_{3} m_{4}}\right) v_{8} \\
v_{3}=\frac{\omega \phi \beta_{v} S_{v}^{0}}{S_{h}^{0}}\left(\frac{m_{2} \varphi(1-\sigma)+m_{3} m_{4}}{m_{2} m_{3} m_{4}}\right) v_{8}, v_{4}=\frac{(1-\sigma) \omega \phi \beta_{v} S_{v}^{0}}{m_{3} m_{4} S_{h}^{0}}\left(\varphi_{1}+m_{4}\right) v_{8} v_{5}=\frac{(1-\sigma) \omega \phi \beta_{v} S_{v}^{0}}{m_{4} S_{h}^{0}}
\end{gathered}
$$

By considering only the non-zero components of left eigenvector $v$ and the non-zero second-order partial derivatives at the disease free-equilibrium point, then we have the following

(i) computation of $a$

$$
\begin{aligned}
& \frac{\partial^{2} f_{2}}{\partial x_{1} \partial x_{j}}\left(x_{0}, 0\right)=0 \text { for } \mathrm{j}=1,2, \ldots, 8 \\
& \frac{\partial^{2} f_{2}}{\partial x_{2} \partial x_{j}}\left(x_{0}, 0\right)=0 \text { for } \mathrm{j}=1,2, \ldots, 7 \text { and } \frac{\partial^{2} f_{2}}{\partial x_{2} \partial x_{8}}\left(x_{0}, 0\right)=\frac{\partial^{2} f_{2}}{\partial x_{8} \partial x_{2}}\left(x_{0}, 0\right)=-\frac{(1-\sigma) \mu \omega \phi \beta_{h}}{S_{h}^{0}} \\
& \frac{\partial^{2} f_{2}}{\partial x_{3} \partial x_{j}}\left(x_{0}, 0\right)=0 \text { for } \mathrm{j}=1,2, \ldots, 7 \text { and } \frac{\partial^{2} f_{2}}{\partial x_{3} \partial x_{8}}\left(x_{0}, 0\right)=\frac{\partial^{2} f_{2}}{\partial x_{8} \partial x_{3}}\left(x_{0}, 0\right)=-\frac{\mu \omega \phi \beta_{h}}{S_{h}^{0}} \\
& \frac{\partial^{2} f_{2}}{\partial x_{4} \partial x_{j}}\left(x_{0}, 0\right)=0 \text { for } \mathrm{j}=1,2, \ldots, 7 \text { and } \frac{\partial^{2} f_{2}}{\partial x_{4} \partial x_{8}}\left(x_{0}, 0\right)=\frac{\partial^{2} f_{2}}{\partial x_{8} \partial x_{4}}\left(x_{0}, 0\right)=-\frac{(1-\sigma) \mu \omega \phi \beta_{h}}{S_{h}^{0}} \\
& \frac{\partial^{2} f_{2}}{\partial x_{5} \partial x_{j}}\left(x_{0}, 0\right)=0 \text { for } \mathrm{j}=1,2, \ldots, 7 \text { and } \frac{\partial^{2} f_{2}}{\partial x_{5} \partial x_{8}}\left(x_{0}, 0\right)=\frac{\partial^{2} f_{2}}{\partial x_{8} \partial x_{5}}\left(x_{0}, 0\right)=-\frac{(1-\sigma) \mu \omega \phi \beta_{h}}{S_{h}^{0}}= \\
& \frac{\partial^{2} f_{2}}{\partial x_{6} \partial x_{j}}\left(x_{0}, 0\right)=0 \text { for } j=1,2, \ldots, 7 \text { and } \frac{\partial^{2} f_{2}}{\partial x_{6} \partial x_{8}}\left(x_{0}, 0\right)=\frac{\partial^{2} f_{2}}{\partial x_{8} \partial x_{6}}\left(x_{0}, 0\right)=-\frac{\mu \omega \phi \beta_{h}}{S_{h}^{0}} \\
& \frac{\partial^{2} f_{2}}{\partial x_{7} \partial x_{j}}\left(x_{0}, 0\right)=0 \text { for } \mathrm{j}=1,2, \ldots, 8 \\
& \cdot \frac{\partial^{2} f_{2}}{\partial x_{8} \partial x_{j}}\left(x_{0}, 0\right)=0 \text { for } \mathrm{j}=1,7,8 \\
& \frac{\partial^{2} f_{3}}{\partial x_{1} \partial x_{j}}\left(x_{0}, 0\right)=0 \text { for } \mathrm{j}=1,2, \ldots, 8 \\
& \frac{\partial^{2} f_{3}}{\partial x_{2} \partial x_{j}}\left(x_{0}, 0\right)=0 \text { for } \mathrm{j}=1,2, \ldots, 7 \text { and } \frac{\partial^{2} f_{3}}{\partial x_{2} \partial x_{8}}\left(x_{0}, 0\right)=\frac{\partial^{2} f_{3}}{\partial x_{8} \partial x_{2}}\left(x_{0}, 0\right)=-\frac{(1-\sigma)(1-\mu) \omega \phi \beta_{h}}{S_{h}^{0}} \\
& \frac{\partial^{2} f_{3}}{\partial x_{3} \partial x_{j}}\left(x_{0}, 0\right)=0 \text { for } \mathrm{j}=1,2, \ldots, 7 \text { and } \frac{\partial^{2} f_{3}}{\partial x_{3} \partial x_{8}}\left(x_{0}, 0\right)=\frac{\partial^{2} f_{3}}{\partial x_{8} \partial x_{3}}\left(x_{0}, 0\right)=-\frac{(1-\mu) \omega \phi \beta_{h}}{S_{h}^{0}} \\
& \frac{\partial^{2} f_{3}}{\partial x_{4} \partial x_{j}}\left(x_{0}, 0\right)=0 \text { for } \mathrm{j}=1,2, \ldots, 7 \text { and } \frac{\partial^{2} f_{3}}{\partial x_{4} \partial x_{8}}\left(x_{0}, 0\right)=\frac{\partial^{2} f_{3}}{\partial x_{8} \partial x_{4}}\left(x_{0}, 0\right)=-\frac{(1-\sigma)(1-\mu) \omega \phi \beta_{h}}{S_{h}^{0}} \\
& \frac{\partial^{2} f_{3}}{\partial x_{5} \partial x_{j}}\left(x_{0}, 0\right)=0 \text { for } \mathrm{j}=1,2, \ldots, 7 \text { and } \frac{\partial^{2} f_{3}}{\partial x_{5} \partial x_{8}}\left(x_{0}, 0\right)=\frac{\partial^{2} f_{3}}{\partial x_{8} \partial x_{5}}\left(x_{0}, 0\right)=-\frac{(1-\sigma)(1-\mu) \omega \phi \beta_{h}}{S_{h}^{0}}= \\
& \frac{\partial^{2} f_{3}}{\partial x_{6} \partial x_{j}}\left(x_{0}, 0\right)=0 \text { for } \mathrm{j}=1,2, \ldots, 7 \text { and } \frac{\partial^{2} f_{3}}{\partial x_{6} \partial x_{8}}\left(x_{0}, 0\right)=\frac{\partial^{2} f_{3}}{\partial x_{8} \partial x_{6}}\left(x_{0}, 0\right)=-\frac{(1-\mu) \omega \phi \beta_{h}}{S_{h}^{0}} \\
& \frac{\partial^{2} f_{3}}{\partial x_{7} \partial x_{j}}\left(x_{0}, 0\right)=0 \text { for } \mathrm{j}=1,2, \ldots, 8 \\
& \frac{\partial^{2} f_{3}}{\partial x_{8} \partial x_{j}}\left(x_{0}, 0\right)=0 \text { for } \mathrm{j}=1,7,8 \\
& \frac{\partial^{2} f_{8}}{\partial x_{1} \partial x_{j}}\left(x_{0}, 0\right)=0 \text { for } \mathrm{j}=1,6,7,8 \text { and } \frac{\partial^{2} f_{8}}{\partial x_{1} \partial x_{3}}\left(x_{0}, 0\right)=-\frac{\omega \phi \beta_{v} s_{v}^{0}}{S_{h}^{0}} \text {, } \\
& \frac{\partial^{2} f_{8}}{\partial x_{1} \partial x_{2}}\left(x_{0}, 0\right)=\frac{\partial^{2} f_{8}}{\partial x_{1} \partial x_{4}}\left(x_{0}, 0\right)=\frac{\partial^{2} f_{8}}{\partial x_{1} \partial x_{5}}\left(x_{0}, 0\right)=-\frac{(1-\sigma) \omega \phi \beta_{v} S_{v}^{0}}{S_{h}^{0}}, \\
& \frac{\partial^{2} f_{8}}{\partial x_{2} \partial x_{j}}\left(x_{0}, 0\right)=0 \text { for } \mathrm{j}=1,8 \text { and } \frac{\partial^{2} f_{8}}{\partial x_{2} \partial x_{4}}\left(x_{0}, 0\right)=\frac{\partial^{2} f_{8}}{\partial^{2} x_{2}{ }^{2}}\left(x_{0}, 0\right)=\frac{\partial^{2} f_{8}}{\partial x_{2} \partial x_{5}}\left(x_{0}, 0\right)=-\frac{(1-\sigma) \omega \phi \beta_{v} S_{v}^{0}}{S_{h}^{0}} \\
& \frac{\partial^{2} f_{8}}{\partial x_{2} \partial x_{3}}\left(x_{0}, 0\right)=\frac{\partial^{2} f_{8}}{\partial x_{2} \partial x_{6}}\left(x_{0}, 0\right)=-\frac{-(1-\sigma) \omega \phi \beta_{v} S_{v}^{0}}{S_{h}^{0^{2}}}, \frac{\partial^{2} f_{8}}{\partial x_{2} \partial x_{7}}\left(x_{0}, 0\right)=\frac{(1-\sigma) \omega \phi \beta_{v}}{S_{h}^{0}}
\end{aligned}
$$




$$
\begin{gathered}
\frac{\partial^{2} f_{8}}{\partial x_{3} \partial x_{j}}=0 \text { for } \mathrm{j}=1,8 \text { and } \frac{\partial^{2} f_{8}}{\partial x_{3} \partial x_{4}}\left(x_{0}, 0\right)=\frac{\partial^{2} f_{8}}{\partial x_{3} \partial x_{5}}\left(x_{0}, 0\right)=-\frac{(1-\sigma) \omega \phi \beta_{v} S_{v}^{0}}{S_{h}^{0}}, \frac{\partial^{2} f_{8}}{\partial^{2} x_{3}{ }^{2}}\left(x_{0}, 0\right)=\frac{\partial^{2} f_{8}}{\partial x_{3} \partial x_{6}}=-\frac{\omega \phi \beta_{v} S_{v}^{0}}{S_{h}^{0}} \\
\frac{\partial^{2} f_{8}}{\partial x_{3} \partial x_{1}}=-\frac{\omega \phi \beta_{v} S_{v}^{0}}{S_{h}^{0^{2}}}, \frac{\partial^{2} f_{8}}{\partial x_{3} \partial x_{7}}=\frac{\omega \phi \beta_{v}}{S_{h}^{0}} \\
\frac{\partial^{2} f_{8}}{\partial x_{4} \partial x_{j}}=0 \text { for } \mathrm{j}=1,8 \text { and } \frac{\partial^{2} f_{8}}{\partial^{2} x_{4}{ }^{2}}\left(x_{0}, 0\right)=\frac{\partial^{2} f_{8}}{\partial x_{4} \partial x_{5}}\left(x_{0}, 0\right)=-2 \frac{(1-\sigma)^{2} \omega \phi \beta_{v} S_{v}^{0}}{S_{h}^{0^{2}}}, \frac{\partial^{2} f_{8}}{\partial x_{4} \partial x_{6}}\left(x_{0}, 0\right)=-\frac{(1-\sigma) \omega \phi \beta_{v} S_{v}^{0}}{S_{h}^{0^{2}}} \\
\frac{\partial^{2} f_{8}}{\partial x_{4} \partial x_{7}}\left(x_{0}, 0\right)=-\frac{\omega \phi \beta_{v} S_{v}^{0}}{S_{h}^{0}} \\
\frac{\partial^{2} f_{8}}{\partial x_{5} \partial x_{j}}=0 \text { for } \mathrm{j}=8 \text { and } \frac{\partial^{2} f_{8}}{\partial^{2} x_{5}{ }^{2}}\left(x_{0}, 0\right)=-2 \frac{(1-\sigma)^{2} \omega \phi \beta_{v} S_{v}^{0}}{S_{h}^{0^{2}}}, \frac{\partial^{2} f_{8}}{\partial x_{5} \partial x_{6}}=-\frac{(1-\sigma) \omega \phi \beta_{v} S_{v}^{0}}{S_{h}^{0}}, \frac{\partial^{2} f_{8}}{\partial x_{5} \partial x_{7}}=\frac{(1-\sigma) \omega \phi \beta_{v}}{S_{h}^{0}} \\
\frac{\partial^{2} f_{8}}{\partial x_{6} \partial x_{j}}\left(x_{0}, 0\right)=0 \text { for } \mathrm{j}=1,6,7,8 \\
\frac{\partial^{2} f_{8}}{\partial x_{7} \partial x_{j}}\left(x_{0}, 0\right)=0 \text { for } \mathrm{j}=1,6,7,8 \\
\frac{\partial^{2} f_{8}}{\partial x_{8} \partial x_{j}}\left(x_{0}, 0\right)=0 \text { for } \mathrm{j}=1,2, \ldots, 8
\end{gathered}
$$$$
a=2 v_{2} w_{2} w_{8} \frac{\partial^{2} f_{2}}{\partial x_{2} \partial x_{8}}\left(x_{0}, 0\right)+2 v_{2} w_{3} w_{8} \frac{\partial^{2} f_{2}}{\partial x_{3} \partial x_{8}}\left(x_{0}, 0\right)+2 v_{2} w_{4} w_{8} \frac{\partial^{2} f_{2}}{\partial x_{4} \partial x_{8}}\left(x_{0}, 0\right)+2 v_{2} w_{5} w_{8} \frac{\partial^{2} f_{2}}{\partial x_{5} \partial x_{8}}\left(x_{0}, 0\right)
$$$$
+2 v_{2} w_{6} w_{8} \frac{\partial^{2} f_{2}}{\partial x_{8} \partial x_{8}}\left(x_{0}, 0\right)
$$$$
+2 v_{2} w_{2} w_{8} \frac{\partial^{2} f_{3}}{\partial x_{2} \partial x_{8}}\left(x_{0}, 0\right)+2 v_{2} w_{3} w_{8} \frac{\partial^{2} f_{3}}{\partial x_{3} \partial x_{8}}\left(x_{0}, 0\right)+2 v_{2} w_{4} w_{8} \frac{\partial^{2} f_{3}}{\partial x_{4} \partial x_{8}}\left(x_{0}, 0\right)+2 v_{2} w_{5} w_{8} \frac{\partial^{2} f_{3}}{\partial x_{5} \partial x_{8}}\left(x_{0}, 0\right)+
$$$$
2 v_{2} w_{6} w_{8} \frac{\partial^{2} f_{3}}{\partial x_{8} \partial x_{8}}\left(x_{0}, 0\right)+
$$

$+2 v_{8} w_{1} w_{3} \frac{\partial^{2} f_{8}}{\partial x_{1} \partial x_{3}}\left(x_{0}, 0\right)+2 v_{8} w_{1} w_{2} \frac{\partial^{2} f_{8}}{\partial x_{1} \partial x_{2}}\left(x_{0}, 0\right)+2 v_{8} w_{1} w_{4} \frac{\partial^{2} f_{8}}{\partial x_{1} \partial x_{4}}\left(x_{0}, 0\right)+2 v_{8} w_{1} w_{5} \frac{\partial^{2} f_{8}}{\partial x_{1} \partial x_{5}}\left(x_{0}, 0\right)+\frac{1}{2} v_{8} w_{2}^{2} \frac{\partial^{2} f_{8}}{\partial^{2} x_{2}{ }^{2}}\left(x_{0}, 0\right)+$

$$
\begin{gathered}
2 v_{8} w_{2} w_{3} \frac{\partial^{2} f_{8}}{\partial x_{2} \partial x_{7}}\left(x_{0}, 0\right)+2 v_{8} w_{2} w_{4} \frac{\partial^{2} f_{8}}{\partial x_{2} \partial x_{4}}\left(x_{0}, 0\right)+2 v_{8} w_{2} w_{5} \frac{\partial^{2} f_{8}}{\partial x_{2} \partial x_{5}}\left(x_{0}, 0\right)+2 v_{8} w_{2} w_{6} \frac{\partial^{2} f_{8}}{\partial x_{2} \partial x_{6}}\left(x_{0}, 0\right)+ \\
2 v_{8} w_{2} w_{7} \frac{\partial^{2} f_{8}}{\partial x_{2} \partial x_{7}}\left(x_{0}, 0\right)+ \\
2 v_{8} w_{3} w_{1} \frac{\partial^{2} f_{8}}{\partial x_{3} \partial x_{1}}\left(x_{0}, 0\right)+2 v_{8} w_{3} w_{4} \frac{\partial^{2} f_{8}}{\partial x_{3} \partial x_{4}}\left(x_{0}, 0\right)+2 v_{8} w_{3} w_{5} \frac{\partial^{2} f_{8}}{\partial x_{3} \partial x_{5}}\left(x_{0}, 0\right)+2 v_{8} w_{3} w_{7} \frac{\partial^{2} f_{8}}{\partial x_{3} \partial x_{7}}\left(x_{0}, 0\right) \\
+\frac{1}{2} v_{8} w_{4}{ }^{2} \frac{\partial^{2} f_{8}}{\partial^{2} x_{4}{ }^{2}}\left(x_{0}, 0\right) \\
+2 v_{8} w_{4} w_{5} \frac{\partial^{2} f_{8}}{\partial x_{4} \partial x_{5}}\left(x_{0}, 0\right)+2 v_{8} w_{4} w_{6} \frac{\partial^{2} f_{8}}{\partial x_{4} \partial x_{6}}\left(x_{0}, 0\right)+2 v_{8} w_{4} w_{7} \frac{\partial^{2} f_{8}}{\partial x_{4} \partial x_{7}}\left(x_{0}, 0\right)+\frac{1}{2} v_{8} w_{5}{ }^{2} \frac{\partial^{2} f_{8}}{\partial^{2} x_{5}{ }^{2}}\left(x_{0}, 0\right)+2 v_{8} w_{5} w_{6} \frac{\partial^{2} f_{8}}{\partial x_{5} \partial x_{6}}\left(x_{0}, 0\right)+ \\
2 v_{8} w_{5} w_{7} \frac{\partial^{2} f_{8}}{\partial x_{5} \partial x_{7}}\left(x_{0}, 0\right) \\
a=\frac{\omega^{2} \phi^{2} w_{8}^{2} v_{8} \beta_{h} \beta_{v} S_{v}}{m_{1} m_{2} m_{3} m_{4} S_{h}}(\Psi-\Gamma)
\end{gathered}
$$

Where,

$$
\begin{gathered}
\Psi=\frac{A_{0}}{\mu_{h}}\left(1-\frac{F_{7}}{m_{1} m_{2} m_{3} m_{4} m}\right) \\
\Gamma=\frac{\omega \phi \beta_{h} F_{1}}{m_{1} m_{2} m_{3} m_{4} S_{h}}\left(m F_{0}+F_{2}\right)+m_{2}\left[m_{3} m_{4} F_{3}+\alpha_{h}\left(m_{4} F_{5}+\varphi_{1} F_{6}\right)\right] \mu+m_{1} m_{3}\left(m_{4} F_{4}+\varphi F_{6}\right)(1-\mu) \\
F_{0}=m_{2}(1-\sigma)\left(m_{3} m_{4}+\alpha_{h}\left(m_{4}+\varphi_{1}\right)\right) \mu+m_{1} m_{3}\left(m_{4}+\varphi(1-\sigma)\right)(1-\mu)>0
\end{gathered}
$$




$$
\begin{gathered}
F_{1}=m_{2} \alpha_{h}(1-\sigma)\left(m_{4}+\varphi_{1}\right) \mu+m_{1} m_{3}\left(m_{3} m_{4}+m_{2} \varphi(1-\sigma)\right)(1-\mu)>0 \\
F_{2}=m_{2} \alpha_{h}\left(\gamma_{1} m_{4}+\gamma_{2} \varphi_{1}\right) \mu+m_{1} m_{3}\left(\gamma m_{4}+\gamma_{2} \varphi\right)(1-\mu)>0 \\
F_{3}=\frac{\omega \phi \beta_{h}}{m_{1} m_{2} m_{3} m_{4} m}\left[m A_{0}+m_{2} \alpha_{h}(1-\sigma)\left(\gamma_{1} m_{4}+\gamma_{2} \varphi_{1}\right) \mu+m_{1} m_{3}\left(\gamma m_{4}+\gamma_{2} \varphi\right)(1-\mu)\right]+\frac{(1-\sigma) m_{5}}{\mu_{v} S_{v}}>0 \\
F_{5}=\frac{\omega \phi \beta_{h}}{m_{1} m_{2} m_{3} m_{4}}\left[m_{2}\left[m(1-\sigma)\left(m_{4}+\alpha_{h} \varphi_{1}\right)+\alpha_{h}\left(\gamma_{1} m_{4}+\gamma_{2} \varphi_{1}\right)\right] \mu+m_{1} m_{3}\left(\gamma m_{4}+\gamma_{2}+m\left((1-\sigma) \varphi+m_{4}\right)\right)(1-\mu)\right]>0 \\
F_{6}=\frac{(1-\sigma) \omega \phi \beta_{h}}{m m_{1} m_{2} m_{2} m_{3} m_{4}}\left[m_{2} \alpha_{h}\left[\left(2(1-\sigma)+\gamma_{1}\right) \varphi_{1}+m_{4}\left(2(1-\sigma)+\gamma_{1}\right)\right] \mu+(1-\mu) m_{1} m_{3}\left[\gamma m+\varphi\left(2(1-\sigma)+\gamma_{2}\right)\right]\right]+\frac{m_{5}}{\mu_{v} S_{v}}>0 \\
\left.F_{7}=\omega \phi \beta_{h} \theta\left[m_{2} \alpha_{h}\left(\gamma_{1} m_{4}+\gamma_{2} \varphi_{1}\right) \mu+m_{1} m_{3}\right] \mu+(1-\mu) m_{1} m_{3}\left[\gamma m+\left(\varphi\left(m+\gamma_{4}\right)+\gamma m_{4}\right)\right]\right]+\frac{(1-\sigma) m_{5} S_{h}{ }^{0}}{\mu_{v} S_{v}{ }^{0}}>0
\end{gathered}
$$

(ii) Computation of $b$

$$
\begin{gathered}
\frac{\partial^{2} f_{2}}{\partial x_{i} ð \beta_{h}}\left(x_{0}, 0\right)=0 \text { for } \mathrm{i}=1,2, \ldots, 7 \text { and } \frac{\partial^{2} f_{2}}{\partial x_{8} ð \beta_{h}}\left(x_{0}, 0\right)=\mu \omega \phi \\
\frac{\partial^{2} f_{3}}{\partial x_{i} \partial \beta_{h}}\left(x_{0}, 0\right)=0 \text { for } \mathrm{i}=1,2, \ldots, 7 \text { and } \frac{\partial^{2} f_{3}}{\partial x_{8} \partial \beta_{h}}\left(x_{0}, 0\right)=(1-\mu) \omega \phi \\
b=v_{2} w_{8} \frac{\partial^{2} f_{2}}{\partial x_{8} \partial \beta_{h}}\left(x_{0}, 0\right)+v_{3} w_{8} \frac{\partial^{2} f_{3}}{\partial x_{8} \partial \beta_{h}}\left(x_{0}, 0\right) \\
=\frac{\omega^{2} \phi^{2} w_{8} v_{8} \beta_{v} S_{v}}{m_{1} m_{2} m_{3} m_{4} S_{h}}\left[m_{2}(1-\sigma)\left(m_{3} m_{4}+\alpha_{h}\left(m_{4}+\varphi_{1}\right)\right) \mu+(1-\mu) m_{1}\left[m_{3} m_{4}+\varphi m_{2}(1-\sigma)\right]\right]>0
\end{gathered}
$$

Theorem 5: If $\Psi>0$ and $\Psi>\Gamma$, then $a>0$ and $\Psi<$ 0 ensures that $a<0$. If $a>0$ and $b>0$, then the model system (1) undergo a backward bifurcation at $R_{0}=1$, otherwise it will exhibit a forward bifurcation. Hence the endemic equilibrium $E^{* *}$ is locally asymptotically stable.

\section{Analysis of the Model with Optimal Control}

In this section, on model system (1) we also incorporate four time dependent control measures namely, (i) the use of insecticide treated bed net (ITN) $\mathrm{u}_{1}(\mathrm{t})=\mathrm{u}_{1}$ as preventive measure i.e., to reduce the number of bites from mosquitoes as they physically provide a barrier between the infectious mosquitoes and the susceptible humans, and also to reduce the population of the mosquitoes by killing them after they land on the treated net. (ii) the effort of screening of quarantined individuals $\mathrm{u}_{2}(\mathrm{t})=\mathrm{u}_{2}$, which helps them to identify whether or not they are with disease symptom, (iii) treatment with drugs $\mathrm{u}_{3}(\mathrm{t})=\mathrm{u}_{3}$, treating individuals who developed symptoms of the disease, and (iv) the use of Indoor Residual Spray (IRS), $\mathrm{u}_{4}(\mathrm{t})=\mathrm{u}_{4}$ as preventive measure i.e., insecticide spray on the breeding site of mosquitoes reduces the number of mosquito populations by killing these rest indoors after feeding.

After incorporating the above stated controls in to the basic model system (1) we get the following modified state equations:

$$
\begin{gathered}
\frac{d S_{h}}{d t}=\Lambda_{h}++\theta R_{h}-\left(\left(1-u_{1}\right) \lambda_{h}+\mu_{h}\right) S_{h} \\
\frac{d E_{q}}{d t}=\left(1-u_{1}\right) \mu \lambda_{h} S_{h}-\left(\alpha_{h}+u_{2}\right) E_{q}-\mu_{h} E_{q} \\
\frac{d I_{h}}{d t}=\left(1-u_{1}\right)(1-\mu) \lambda_{h} S_{h}-\left(\varphi+1-u_{2}\right) I_{h}-\left(\mu_{h}+\delta_{h}+\gamma\right) I_{h} \\
\frac{d I_{q}}{d t}=\left(\alpha_{h}+u_{2}\right) E_{q}-\left(\varphi_{1}+\left(1-u_{2}\right)\right) I_{q}-\left(\mu_{h}+\delta_{h}+\gamma_{2}\right) I_{q} \\
\frac{d H_{p}}{d t}=\left(\varphi+\left(1-u_{2}\right)\right) I_{h}+\left(\varphi_{1}+\left(1-u_{2}\right)\right) I_{q}-\left(\gamma_{1}+\tau u_{3}\right) H_{p}-\left(\mu_{h}+\delta_{h}\right) H_{p} \\
\frac{d R_{h}}{d t}=\gamma I_{h}+\gamma_{2} I_{q}+\left(\gamma_{1}+\tau u_{3}\right) H_{p}-\left(\theta+\mu_{h}\right) R_{h}
\end{gathered}
$$




$$
\begin{gathered}
\frac{d S_{v}}{d t}=\Lambda_{v}-\left(\left(1-u_{1}\right) \lambda_{v}+\mu_{v}+\delta u_{1}+\beta u_{4}\right) S_{v} \\
\frac{d I_{v}}{d t}=\left(1-u_{1}\right) \lambda_{v} S_{v}-\left(\mu_{v}+\delta_{v}+\delta u_{1}+\beta u_{4}\right) I_{v}
\end{gathered}
$$

The purpose of the study of endemic malaria disease model system (1) with optimal control is to minimize the numbers of Infected non-quarantined, hospitalized (infected isolated) humans, and infected mosquitoes and also increase the number of recovered humans furthermore, explore the best combinations of the strategy in malaria control and elimination. For this end, its objective function is defined based on the approach [28]. Thus, the objective function of (17) is

$$
J\left(u_{1}, \quad u_{2}, \quad u_{3}, u_{4}\right)=\int_{0}^{t_{f}}\left(d_{1} E_{q}+d_{2} I_{h}+d_{3} I_{q}+d_{4} H_{p}+d_{5} I_{v}+\frac{\pi_{1}}{2} u_{1}^{2}+\frac{\pi_{2}}{2} u_{2}^{2}+\frac{\pi_{3}}{2} u_{3}^{2}+\frac{\pi_{4}}{2} u_{4}^{2}\right) d t
$$

Where, $d_{1}, d_{2}, d_{3}, d_{4}$, and $d_{5}$, are the balancing cost factors due to scale and $\pi_{1}, \pi_{2}, \pi_{3}$, and $\pi_{4}$ denote the weighting constants for making uses of control strategies using $u_{1}, u_{2}, u_{3}$, and $u_{4}$ controls. Consequently, we attempt to expect an optimal control $u_{1}^{*}, u_{2}^{*}, u_{3}^{*}$ and $u_{4}^{*}$ such that

$$
J\left(u_{1}^{*}, u_{2}^{*}, u_{3^{\prime}}^{*}, u_{4^{*}}^{*}\right)=\operatorname{minJ}\left(u_{1}, u_{2}, u_{3}, u_{4}\right), U=\left\{\left(u_{1}, u_{2}, u_{3}, u_{4}\right): 0 \leq u_{i} \leq 1, i=1,2,3,4\right\}
$$

The Hamiltonian $\mathrm{H}$, associated with problems (17) - (19) is

$$
\begin{gathered}
H=\left(S_{h}, E_{q}, I_{h}, I_{q}, H_{p}, R_{h}, S_{v}, I_{v}, t\right)= \\
L\left(E_{q}, I_{h}, I_{q}, H_{p}, I_{v}, u_{1}, u_{2}, u_{3}, u_{4}, t\right)+\lambda_{1} \frac{d S_{h}}{d t}+\lambda_{2} \frac{d E_{q}}{d t}+\lambda_{3} \frac{d I_{h}}{d t}+\lambda_{4} \frac{d I_{q}}{d t}+\lambda_{5} \frac{d H_{p}}{d t}+ \\
\lambda_{6} \frac{d R_{h}}{d t}+\lambda_{7} \frac{d S_{v}}{d t}+\lambda_{8} \frac{d I_{v}}{d t}
\end{gathered}
$$

Where,

$$
L\left(E_{q}, I_{h}, I_{q}, H_{p}, I_{v}, u_{1}, u_{2}, u_{3}, u_{4}, t\right)=d_{1} E_{q}+d_{2} I_{h}+
$$

$d_{3} I_{q}+d_{4} H_{p}+d_{5} I_{v}+\frac{1}{2} \sum_{1}^{4} \pi_{i} u_{i}^{2}$, for $\mathrm{i}=1,2,3,4$ and $\lambda_{i}$, for $\mathrm{i}=1,2,3,4,5,6,7,8$ are adjoint variable of functions to be determined. The optimal control must satisfy the necessary conditions that is emanated from the Pontryagins's Maximum Principle [27, 28]. This concept transpose (1) and (17) into a type of problem characterized with minimizing pointwise the Hamiltonian $\mathrm{H}$ respect to

$$
\frac{\mathrm{d} \lambda_{\mathrm{i}}}{\mathrm{dt}}=-\frac{\partial \mathrm{H}}{\partial \mathrm{i}}
$$

Where $\mathrm{i}=1,2,3,4,5,6,7,8$ and with transversality conditions

$$
\lambda_{i}\left(t_{f}\right)=0 \text { for } i=1,2,3,4,5,67,8
$$

Proposition 1: The optimal control $\left(u_{1}^{*}, u_{2}^{*}, u_{3}^{*}, u_{4}^{*}\right)$ that minimize the objective function over $U$ is given by

$$
\begin{gathered}
\frac{\partial \mathrm{H}}{\partial \mathrm{u}_{i}}=0, \text { at } \mathrm{u}_{i}=u_{i}^{*} \text { where } i=1,2,3,4: \\
u_{1}^{*}=\min \left\{\max \left(0, \frac{S_{h}^{*} \lambda_{h}^{*}\left[\left(\lambda_{3}-\lambda_{1}\right)+\mu\left(\lambda_{2}-\lambda_{3}\right)\right]+\lambda_{v}^{*} S_{v}^{*}\left(\lambda_{7}-\lambda_{8}\right)+\delta\left(S_{v}^{*} \lambda_{7}+I_{v}^{*} \lambda_{8}\right)}{\pi_{1}}\right), 1\right\} \\
\left.u_{2}^{*}=\min \left\{\max \left(0, \frac{\varphi I_{h}\left(\lambda_{5}-\lambda_{3}\right)+\varphi_{1} I_{q}\left(\lambda_{5}-\lambda_{4}\right)+\left(\alpha \lambda_{4}+\lambda_{2}\right) E_{q}^{*}}{\pi_{2}}\right), 1\right)\right\} \\
\left.u_{3}^{*}=\min \left\{\max \left(0, \frac{H_{p}^{*} \gamma_{1} \tau\left(\lambda_{6}-\lambda_{5}\right)}{\pi_{3}}\right), 1\right)\right\} \\
\left.u_{4}^{*}=\min \left\{\max \left(0, \frac{\beta\left(\lambda_{7} S_{v}^{*}+\lambda_{8} I_{v}^{*}\right)}{\pi_{4}}\right), 1\right)\right\}
\end{gathered}
$$

Proof:

From theorem 1 and 2 above, boundedness and positivity of the state solutions respectively are shown. From [29, 30], the condition of possible existence of an optimal control is based on the convexity of the integrand of $J\left(u_{1}, u_{2}, u_{3}, u_{4}\right)$ with respect to $u_{1}, u_{2}, u_{3}$ and $u_{4}$, and Lipschitz property of the state system with respect to state variables. The Hamiltonian function determines at the optimal control level leads to the adjoint variables. Thus, the adjoint equations can be rearranged as 


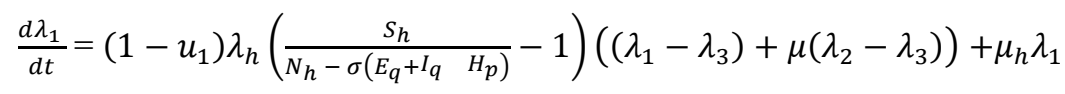

$$
\begin{aligned}
& \frac{d \lambda_{2}}{d t}=-d_{1}++\frac{\left(1-u_{1}\right)(1-\sigma)}{N_{h}-\sigma\left(E_{q}+I_{q} \quad H_{p}\right)}\left[\lambda_{h} S_{h}\left[\left(\lambda_{1}-\lambda_{3}\right)+\mu\left(\lambda_{2}-\lambda_{3}\right)\right]+\lambda_{v} S_{v}\left(\lambda_{7}-\lambda_{8}\right)\right] \\
& +\mu_{h} \lambda_{2}+\left(\alpha_{h}+u_{2}\right)\left(\lambda_{2}-\lambda_{4}\right) \\
& \frac{d \lambda_{3}}{d t}=-d_{2}+\gamma\left(\lambda_{3}-\lambda_{6}\right)+\left(\varphi+\left(1-u_{2}\right)\right)\left(\lambda_{3}-\lambda_{5}\right)+\left(\mu_{h}+\delta_{h}\right) \lambda_{3}+\frac{\left(1-u_{1}\right)(1-\sigma) \lambda_{v} S_{v}}{N_{h}-\sigma\left(E_{q}+I_{q} \quad H_{p}\right)}\left(\phi \omega \beta_{v}-\lambda_{v}\right)\left(\lambda_{7}-\lambda_{8}\right)
\end{aligned}
$$

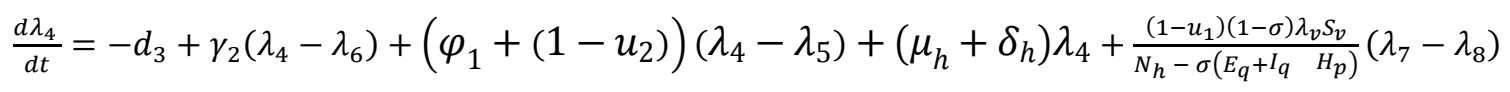

$$
\begin{aligned}
& \frac{d \lambda_{5}}{d t}=-d_{4}+\left(\gamma_{1}-\tau u_{3}\right)\left(\lambda_{5}-\lambda_{6}\right)+\left(\mu_{h}+\delta_{h}\right) \lambda_{5}+\frac{\left(1-u_{1}\right)(1-\sigma) \lambda_{v} S_{v}}{N_{h}-\sigma\left(E_{q}+I_{q} H_{p}\right)}\left(\lambda_{7}-\lambda_{8}\right) \\
& \frac{d \lambda_{6}}{d t}=\mu_{h} \lambda_{6}+\theta\left(\lambda_{6}-\lambda_{1}\right) \\
& \frac{d \lambda_{7}}{d t}=\left(1-u_{1}\right) \lambda_{v}\left(\lambda_{7}-\lambda_{8}\right)+\left(\mu_{v}+\delta u_{1}+\beta u_{4}\right) \lambda_{7} \\
& \frac{d \lambda_{8}}{d t}=-d_{5}+\left(\mu_{v}+\delta_{v}+\delta u_{1}+\beta u_{4}\right) \lambda_{8}^{*}+\frac{\left(1-u_{1}\right) \phi \omega \beta_{h}}{N_{h}-\sigma\left(E_{q}+I_{q} H_{p}\right)}\left(\left(\lambda_{1}-\lambda_{3}\right)+\mu\left(\lambda_{2}-\lambda_{3}\right)\right)
\end{aligned}
$$

\section{Numerical Simulations Results}

In this section, numerical simulations are performed to illustrate the effects of malaria control measures by applying different control strategies. We apply the parameter values listed in Tables 2 and 3 to obtain numerical results for the optimal system by using a forward-backward iterative method [31].

Initial values that we used for simulation of the optimal control are: $S_{h}(0)=700, E_{q}(0)=250, I_{h}(0)=$ $30, I_{q}(0)=80, H(0)=100, R_{h}(0)=30, S_{v}(0)=5000$, and $I_{v}(0)=100$. And also coefficients of the state and controls are given below. Due to the lack of the available literatures and data, as an example, we have assumed cost coefficients for, $d_{1}=4, d_{2}=2, d_{3}=4, d_{4}=d_{5}=$ $2, \pi_{1}=2, \pi_{2}=4, \pi_{3}=36 \pi_{4}=1$, respectively. And $u_{1}=$ $0.0904, u_{2}(t)=0.0802, u_{3}(t)=0.1650$, and $u_{4}(t)=0.0760$ and maximums of $u_{1}(t), u_{2}(t), u_{3}(t)$ and $u_{4}(t)$ are taken as 1 .

Table 2. Parameter Values model system (1).

\begin{tabular}{lll}
\hline Parameter & Value & Source \\
\hline$\beta_{h}$ & 0.0655 & {$[32]$} \\
$\Lambda_{h}$ & 0.000000104 & Assumed \\
$\mu_{h}$ & 0.00005447 & {$[33]$} \\
$\delta_{h}$ & 0.05 & {$[34]$} \\
$\alpha_{h}$ & 0.07143 & {$[32]$} \\
$\gamma$ & 0.005 & {$[35]$} \\
$\sigma$ & 0.8900 & {$[17]$} \\
$\gamma_{1}$ & 0.05 & Assumed \\
$\gamma_{2}$ & 0.005 & Assumed \\
$\theta$ & 0.01095 & {$[32]$} \\
$\tau$ & 0.5000 & {$[32]$} \\
$\omega$ & 0.2000 & {$[36]$} \\
$\beta_{v}$ & 0.0900 & {$[37]$} \\
$\Lambda_{v}$ & 200 & Assumed \\
$\mu_{v}$ & 0.0400 & {$[38]$} \\
$\delta_{v}$ & 0.0500 & {$[39]$} \\
$\beta$ & 0.2500 & {$[32]$} \\
$\delta$ & 0.2500 & {$[32]$} \\
$\phi$ & 0.5020 & {$[36]$} \\
$\varphi$ & 0.07 & Assumed \\
$\varphi_{1}$ & 0.07 & Assumed \\
$\mu$ & 0.0420 & {$[32]$} \\
\hline
\end{tabular}


Table 3. Prevention and control variables in the model.

\begin{tabular}{llll}
\hline Symbol & Description & Value & Source \\
\hline$u_{1}(t)$ & Preventive measure using insecticide treated bed nets & 0.0904 & {$[32]$} \\
$u_{2}(t)$ & The effort of screening of quarantine individuals & 0.0802 & Assumed \\
$u_{3}(t)$ & The control effort on treatment of infectious individuals & 0.1650 & {$[32]$} \\
$u_{4}(t)$ & Preventing measure using indoor residual spray & 0.0760 & {$[32]$} \\
\hline
\end{tabular}

From case (iii) of theorem 4 above, if $R_{0}>1$, there are only two equilibria and the disease free-equilibrium is unstable and the larger endemic equilibrium is stable. The qualitative bifurcation diagrams describing the two types of bifurcation at $R_{0}=1$ are shown in figure 2 below.

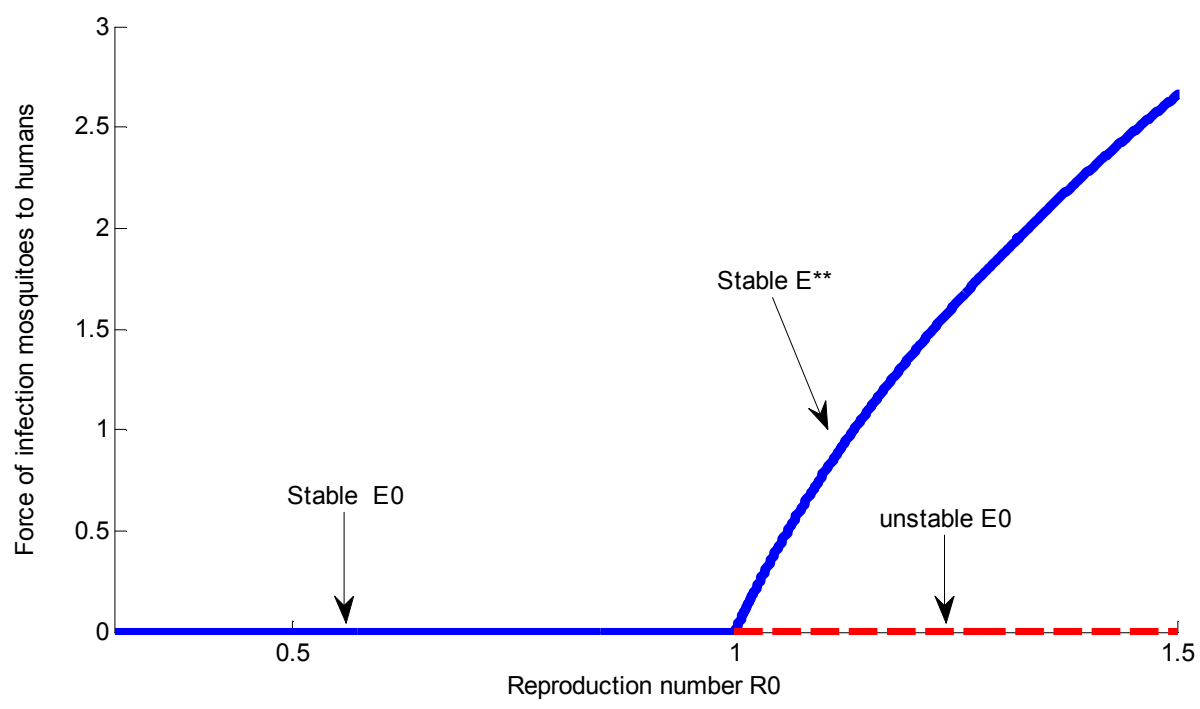

(a)

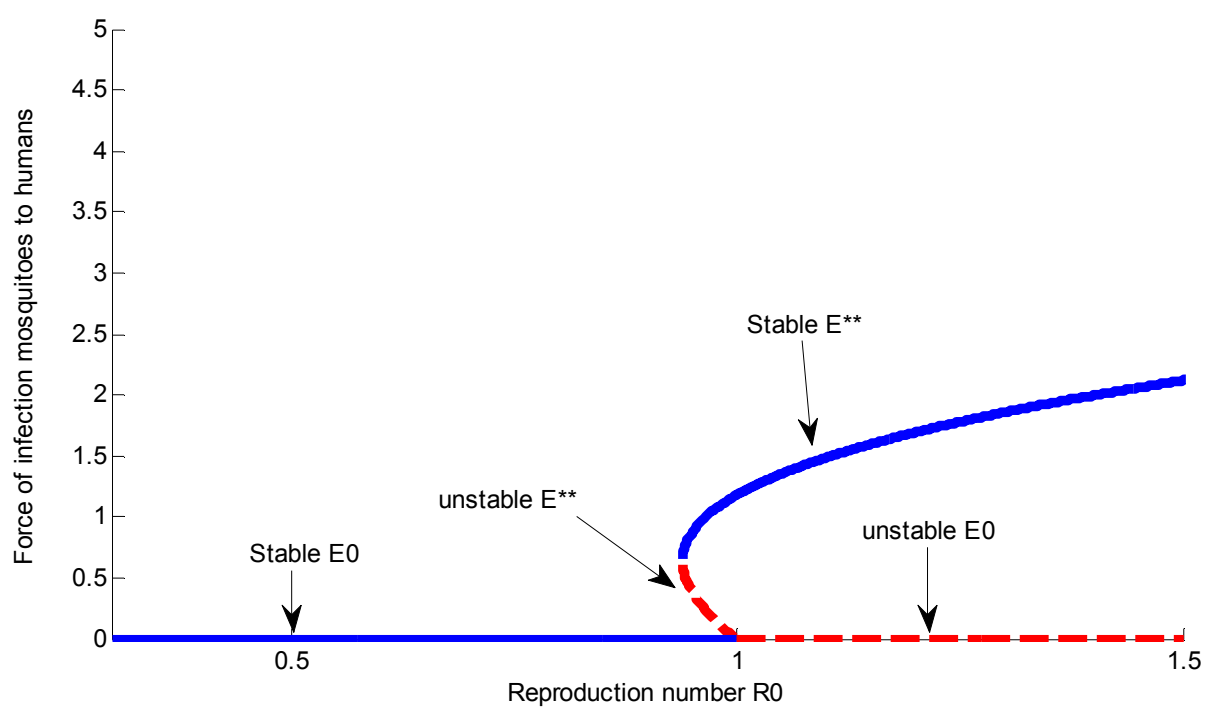

(b)

Figure 2. Qualitative bifurcation diagrams for the forward (a) and backward (b) bifurcations respectively.

Note that, the solid line or blue color denotes both stable disease free equilibrium $E_{0}$ and endemic equilibrium $E^{* *}$ respectively. The dashed line or red color denotes both un stabile disease free equilibrium $E_{0}$ and endemic equilibrium $E^{* *}$ respectively. In the backward bifurcation scenario, if $R_{0}<1$, then the disease control more depends on the initial sizes of the sub populations of the model. Contrary, reducing $R_{0}$ below the saddle node bifurcation value that is, $R_{c}<R_{0}<\min \left(1, R_{-}\right)$or $\max \left(1, R_{+}\right)<R_{C}<R_{0}<1$, may result in disease elimination.

\subsection{Controlling Endemic Malaria Disease Using Imperfect Quarantine Strategy}

In this strategy, we simulated the model system (1) by incorporating imperfect quarantine to reduce the number of susceptible mosquitos' bites from or contacts with malaria infectious humans. 


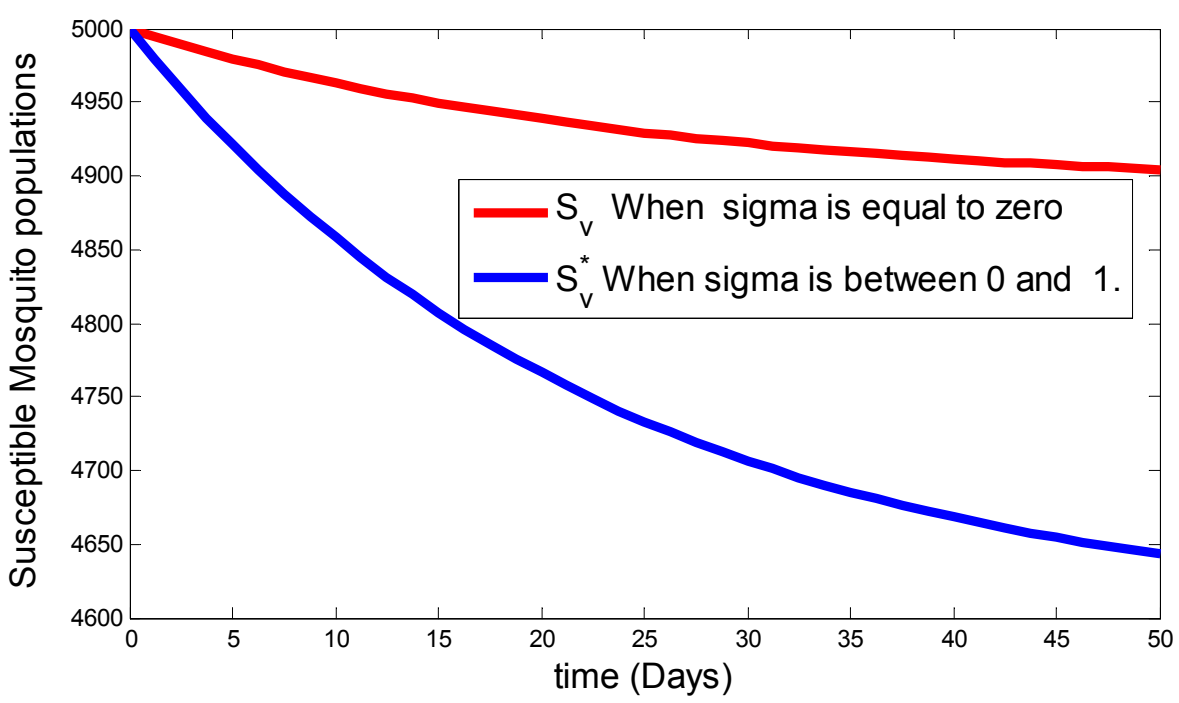

Figure 3. Simulation of endemic malaria model (1) of Susceptible mosquito populations.

Figure 3 above, represents the numbers of susceptible mosquitoes $S_{v}$ during the implementation of the strategy. From the figure, it is clearly seen that the graphs were exponentially decreased and smaller in number at the end of implementation of intervention time above in the case with imperfect quarantine $(0<\sigma<1)$ compered to in case without quarantine $\sigma=0$. From this we conclude that imperfect quarantine strategy plays a great role in reducing the numbers of susceptible mosquitoes bites from or contacts with malaria infectious humans and hence eliminate the spread and transmission of the disease through the human populations.

To examine the impact of the combination of each control and elimination of malaria disease, we used the following strategy:

(i) Implementing ITN $u_{1}(t)$ and screening $u_{2}(t)$ as intervention

(ii) Implementing ITN and IRS as intervention (iii) Implementing screening and treatment as intervention

(iv) Implementing ITN, screening and treatment as intervention

(v) Implementing ITN, treatment and IRS as intervention

(vi) Implementing screening, treatment and IRS as intervention

(vii) Implementing ITN, screening, and IRS as intervention

(viii) Implementing ITN, screening, treatment effort and IRS as intervention

\subsection{Controlling with Insecticide Treated Net ITN and Screening}

In this case, we simulated the model by incorporating optimized Insecticide Treated Net and screening as disease control strategy.

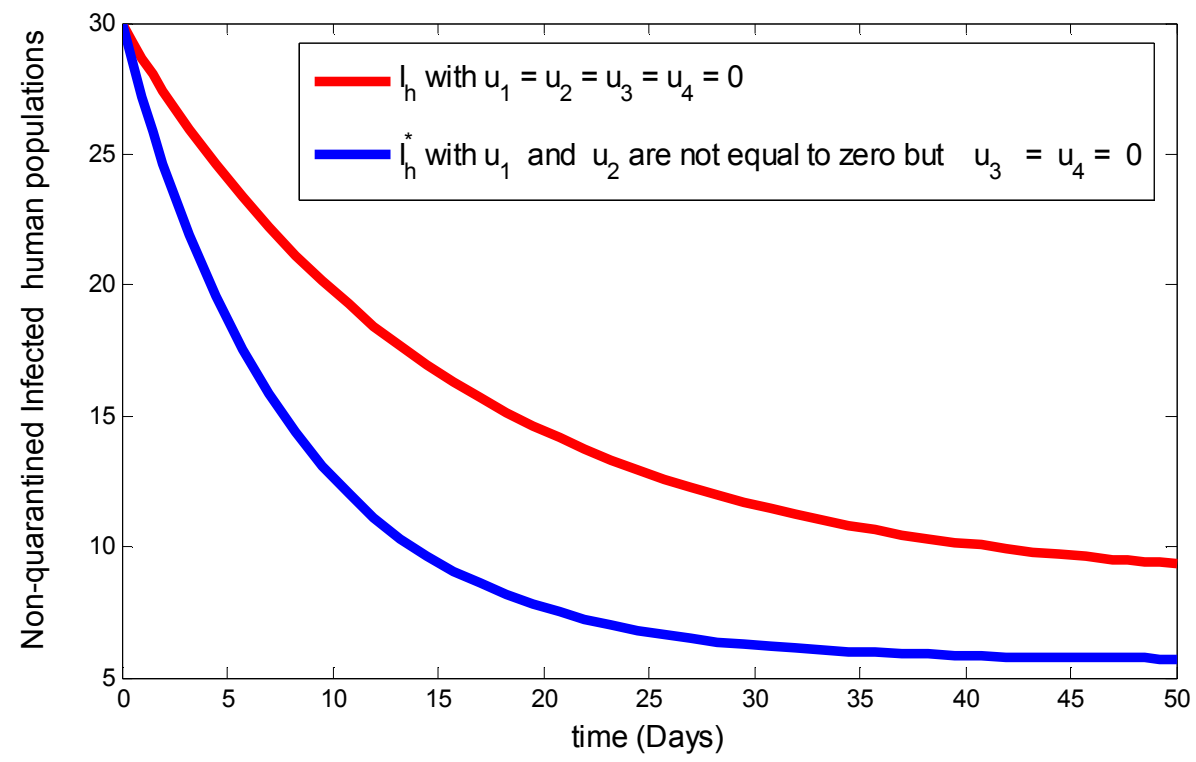

(a) 


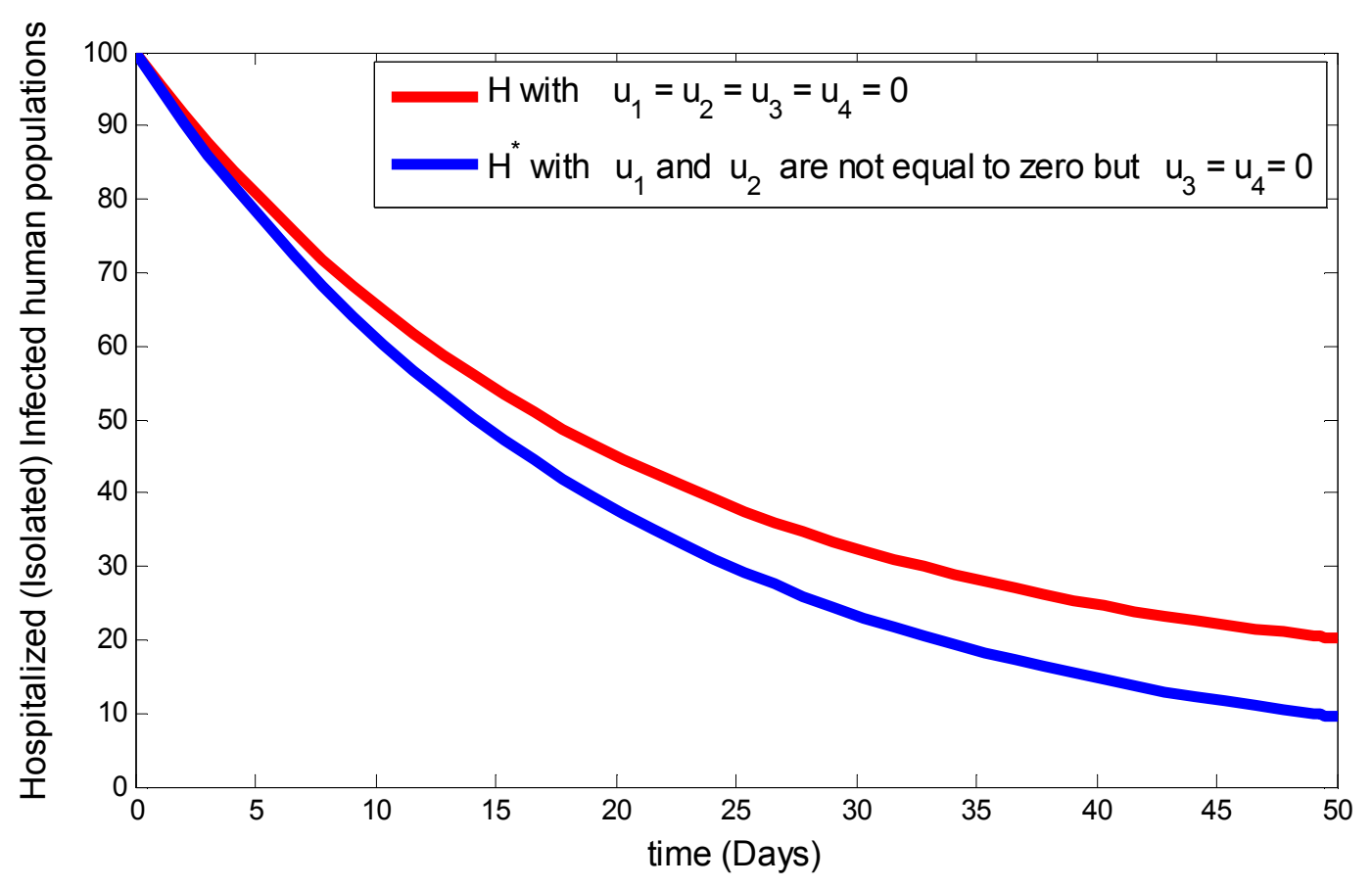

(b)

Figure 4. Simulation of endemic malaria model with ITN and Screening.

In Figue 4 (a) and (b) above, there is a small number difference between the states with control $\left(u_{1}(t) \neq\right.$ $\left.0, u_{2}(t) \neq 0 . u_{3}(t)=u_{4}(t)=0\right)$ represented by blue color and without controls $\left(u_{1}(t)=u_{2}(t)=u_{2}(t)=\right.$ $u_{3}(t)=0$ ) represented by red color. It is clearly seen from the figure that, both the number of infected humans and hospitalized (infected isolated) humans are exponentially decreased with time but their numbers cannot be zero at final time of implementation of the strategy. From this we can conclude that using only the combination of insecticide treated net ITN and screening, it is possible to reduce the number of malaria infectious individuals even without treating asymptomatic individuals.

\subsection{Controlling with Insecticide Treated Net ITN and Indoor Residual Spray IRS}

This case, we simulated the model by incorporating optimized insecticide treated net and Indoor Residual spray IRS as disease control strategy to optimize the objective function $\mathrm{J}$

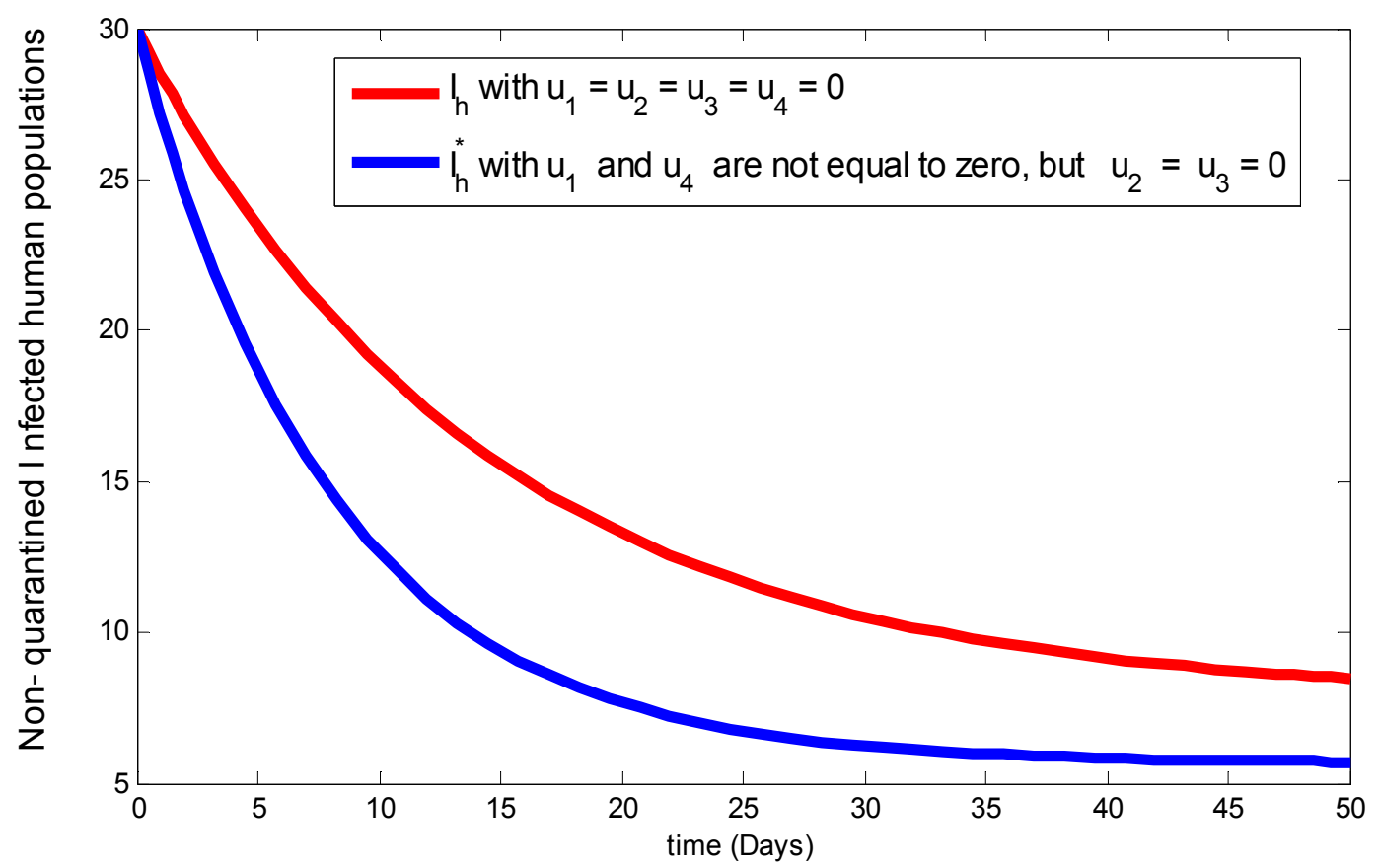

(a) 


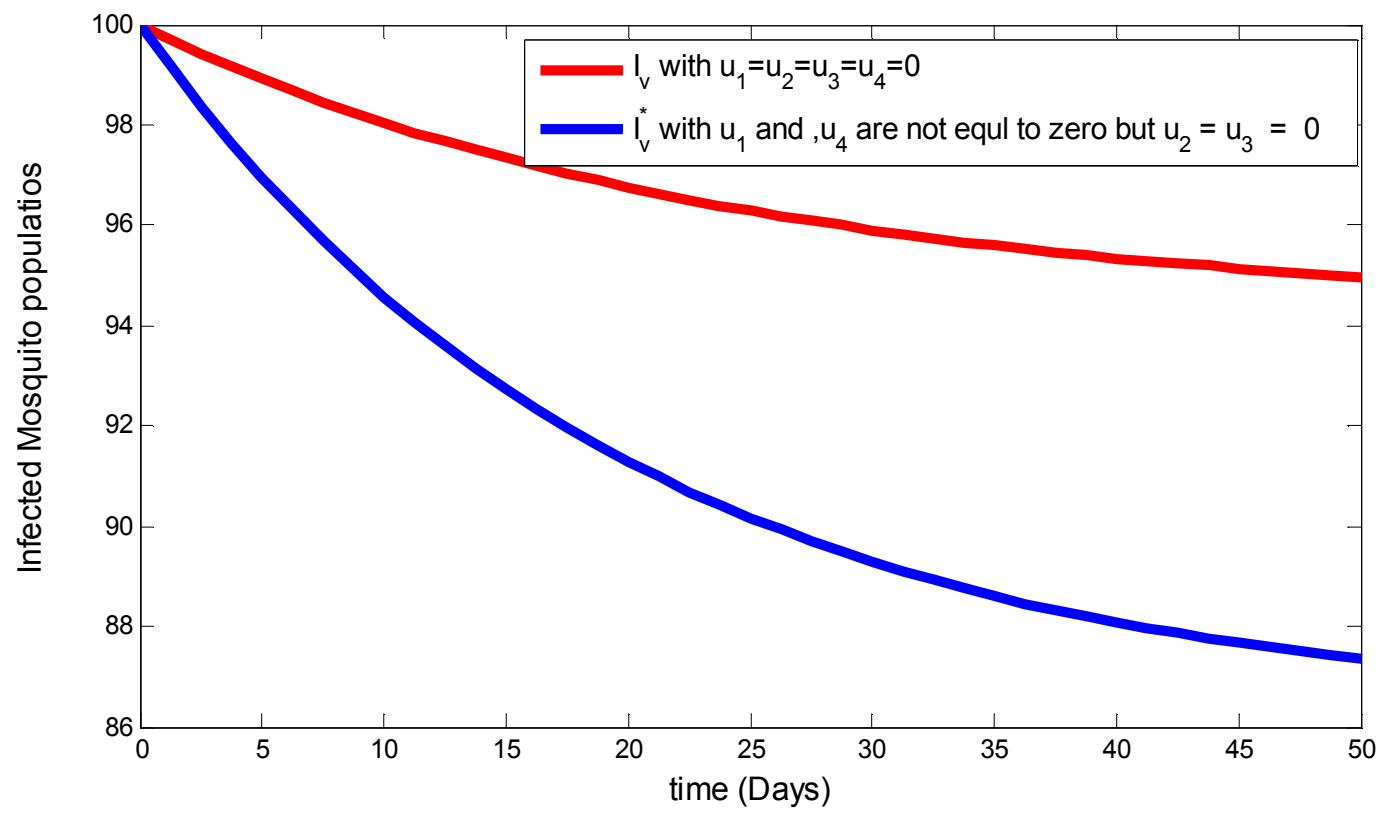

(b)

Figure 5. Simulation of endemic malaria model with ITN and IRS.

Figure 5 (a) and (b), above represents the numbers of infected non-quarantined humans $I_{h}$ and infected mosquitoes $I_{v}$ during the implementation of the strategy. From the figure, it is clearly seen that the numbers of infected non-quarantined humans and infected mosquitoes are smaller in case with control $\left(u_{1}(t) \neq\right.$ $\left.0, u_{4}(t) \neq 0, u_{2}(t)=u_{3}(t)=0\right)$ than in the case without control $\left(u_{1}(t)=u_{2}(t)=u_{2}(t)=u_{3}(t)=0\right)$ at the final time of implementation of the strategy.

\subsection{Control with Screening and Treatment}

In this case, we simulated the model by incorporating optimized screening and treatment as disease control strategy to optimize the objective function $\mathrm{J}$.

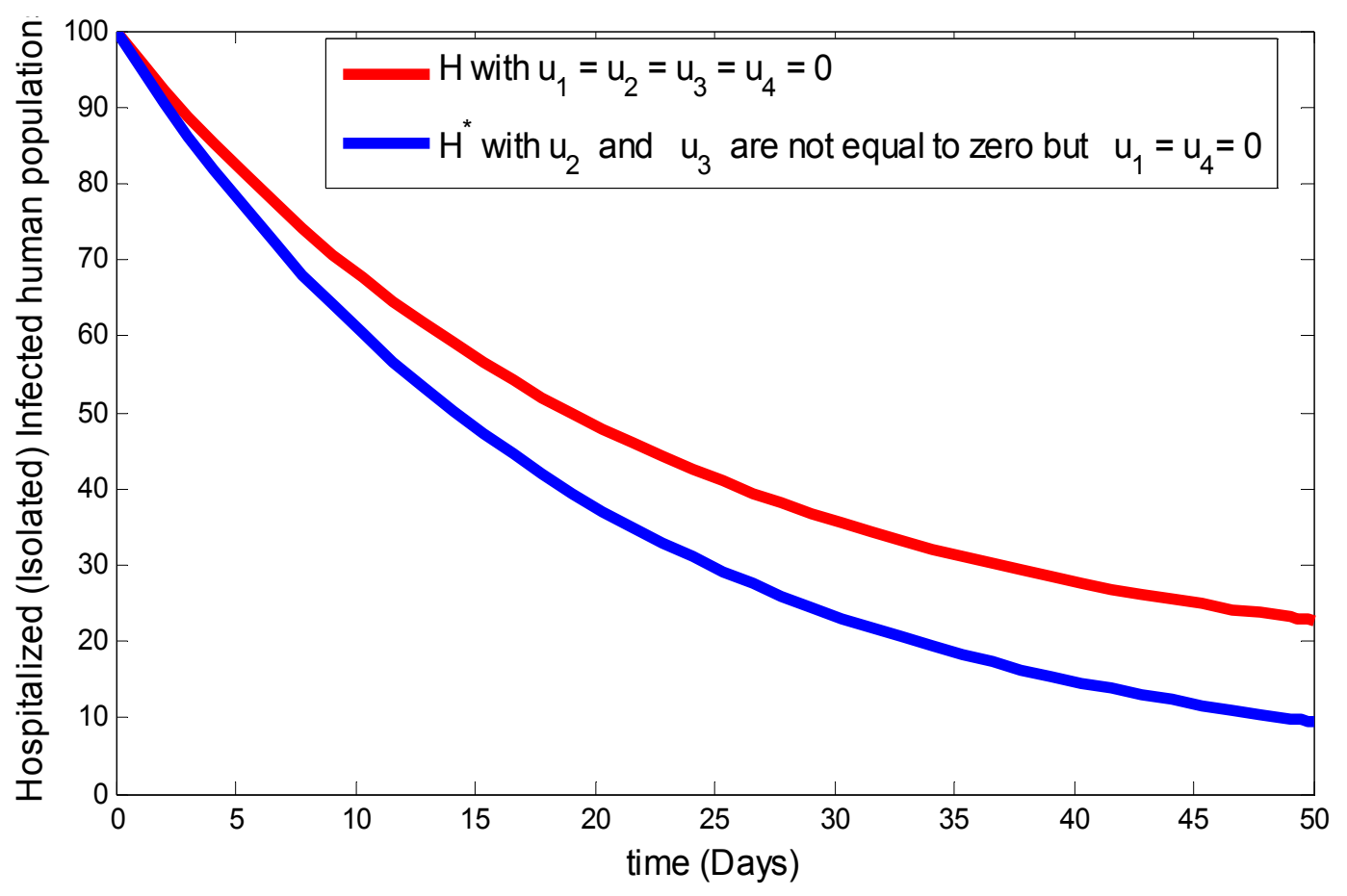




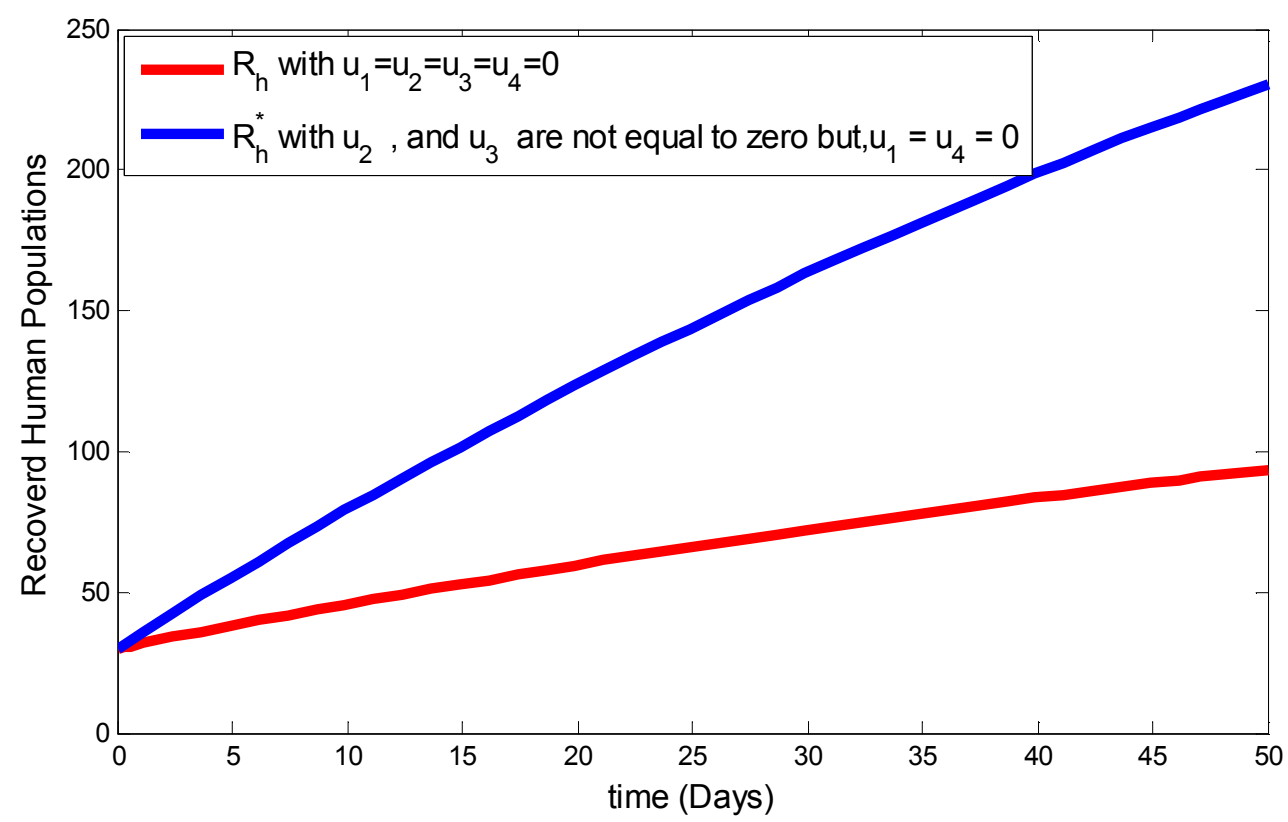

Figure 6. Simulation of endemic malaria model with screening and treatment.

Figure 6 (a) and (b), above represents the numbers of hospitalized (infected isolated) $H_{p}$ humans and recovered $R_{h}$ humans during the implementation of the strategy with control and without control represented by blue and red color respectively. From the figure, it is clearly seen that the numbers of hospitalized (infected isolated) humans are decreased more with time incase with control than without control. Similarly, the number of recovered humans are large incase with control while their numbers are small incase without control at the final time of implementation of the strategy.

\subsection{Control with Preventive Insecticide Treated Net ITN, Screening and Treatment}

In this case, we simulated the model by incorporating optimized insecticide treated net ITN, screening and treatment as disease control strategy to optimize the objective function $\mathrm{J}$.
Figure 7 (a), (b) and (c), represents the numbers of infected non-quarantined $I_{h}$, hospitalized (infected isolated) $H_{p}$ and infected mosquitoes $I_{v}$ during the implementation of the strategy with control and without control represented by blue and red color respectively. From the figure, it is clearly seen that the numbers of infected non-quarantined humans and hospitalized (infected isolated) are smaller at the end of implementation of intervention time above in the case with control than without control. Similarly, the number of infected mosquitoes are large incase without control while their numbers are small incase with control at final time of implementation of the strategy. The reason is that applying optimized the combination of insecticide treated net ITN, screening and treatment only control intervention decreases more the burden of the disease than a combination of two controls intervention but it cannot be eradicate the disease in the community.

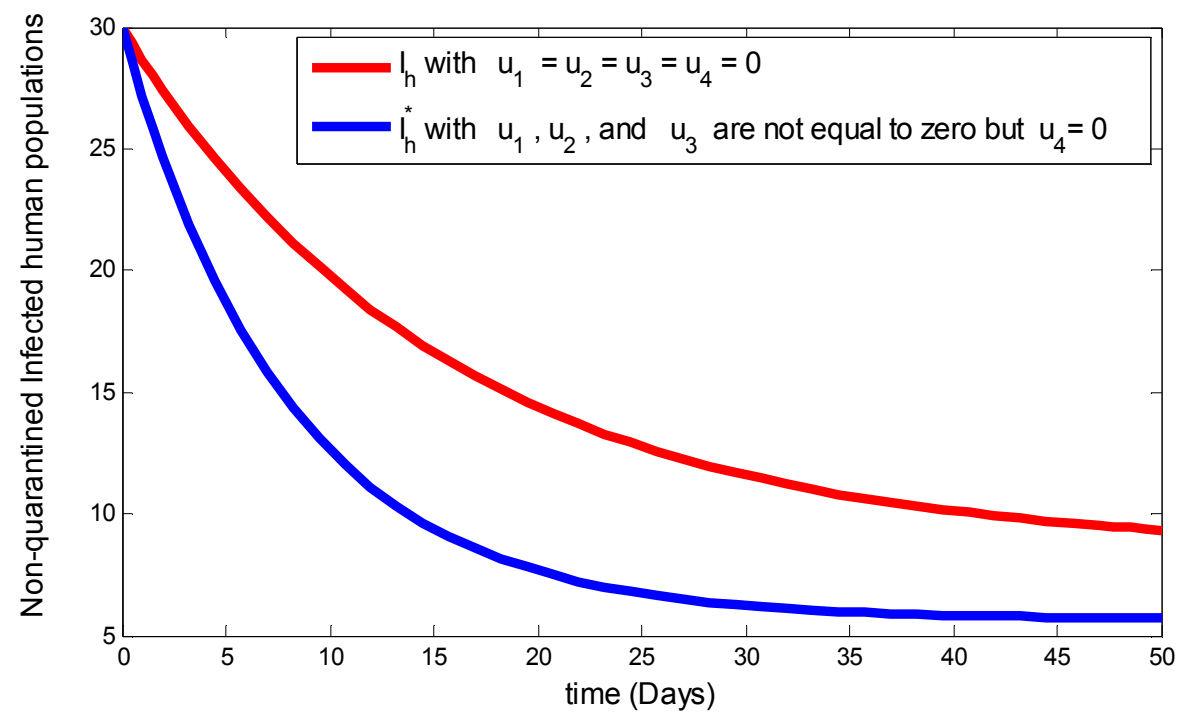

(a) 


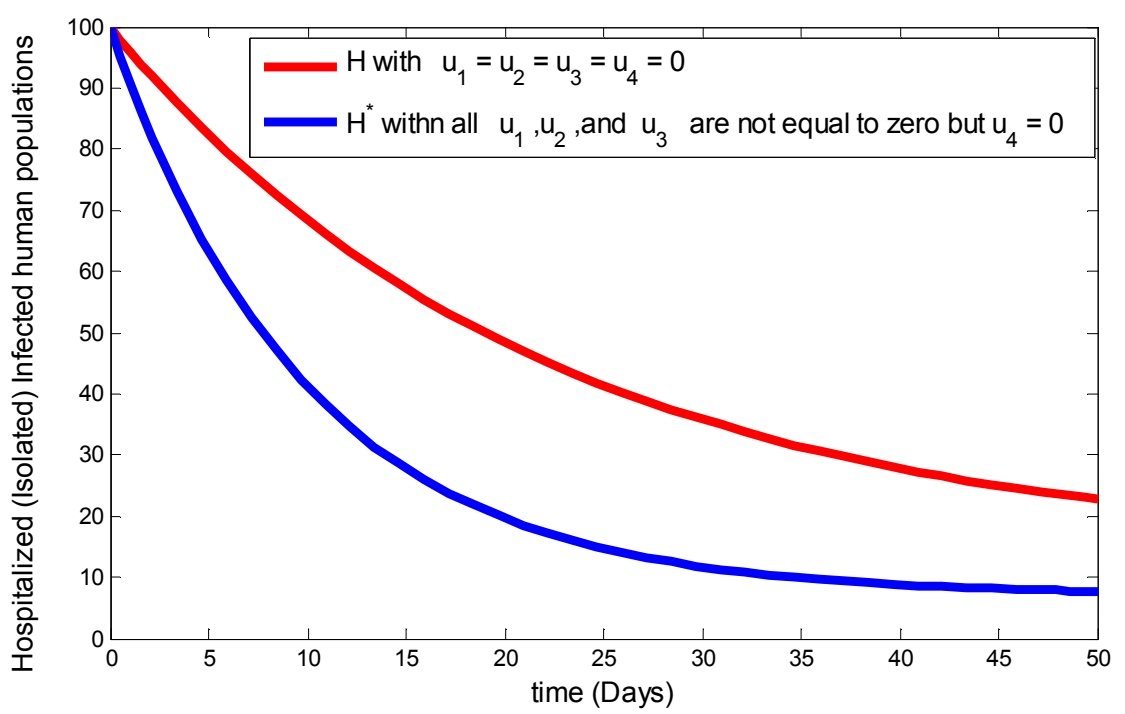

(b)

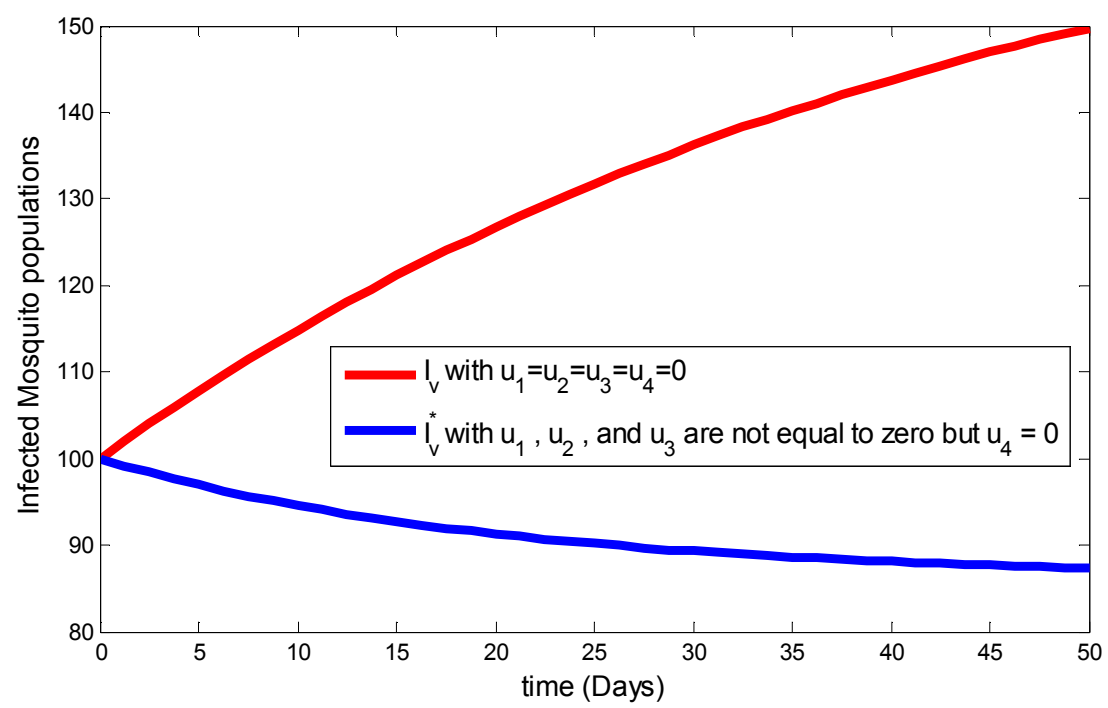

(c)

Figure 7. Simulation of endemic malaria model with screening, treatment and ITN.

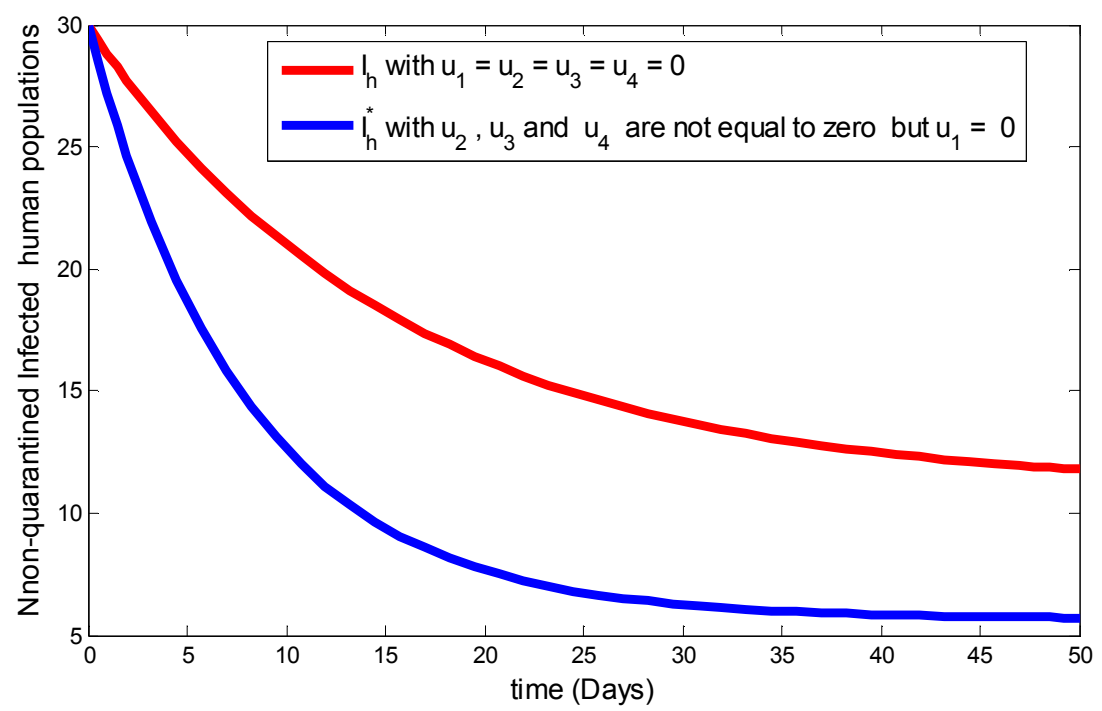

(a) 


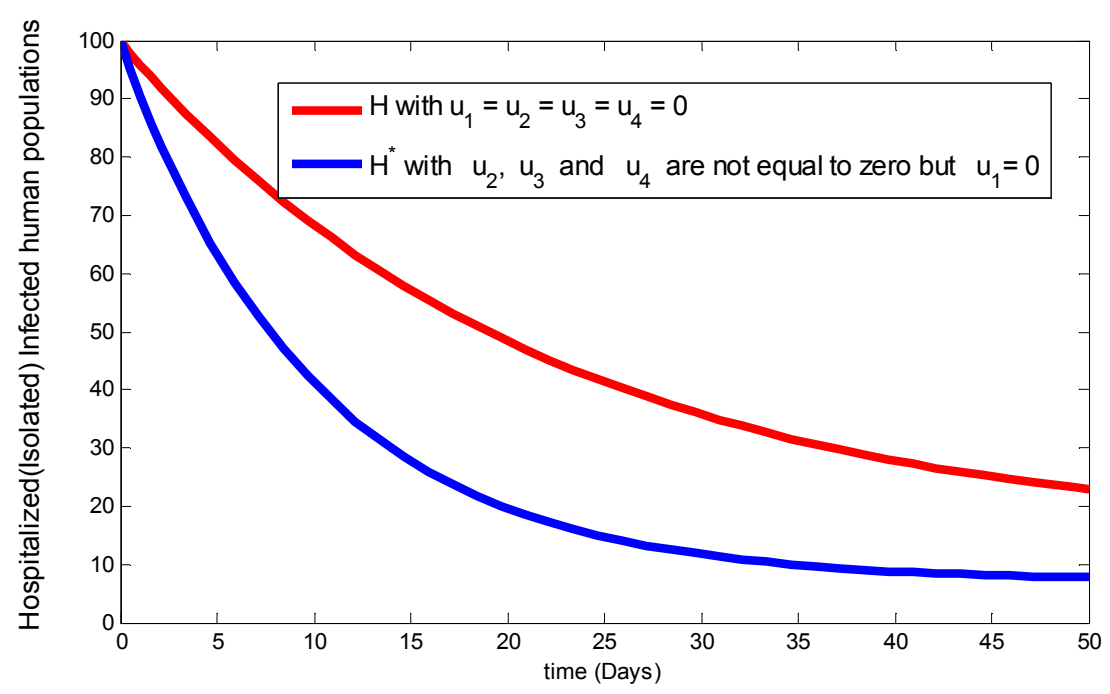

(b)

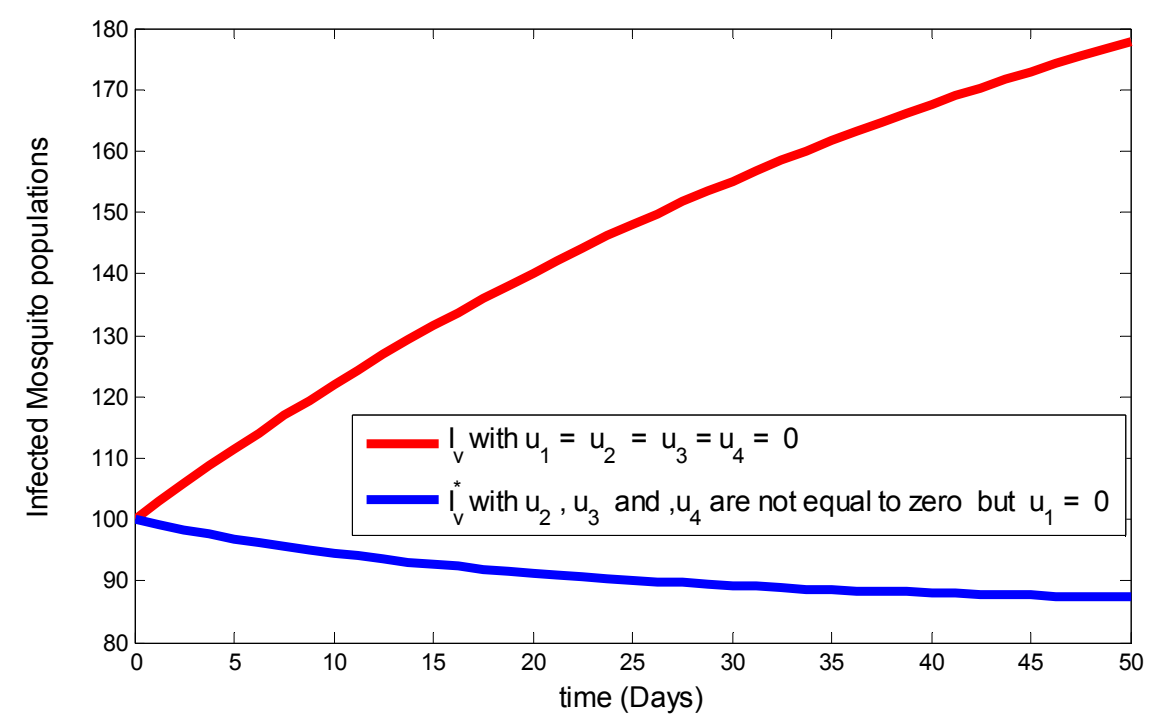

(c)

Figure 8. Simulation of endemic malaria model with Screening, Treatment and IRS.

\subsection{Control with Combination of Screening, Treatment and Indoor Residual Spray IRS}

In this case, we simulated the model by incorporating optimized indoor residual spray IRS, screening and treatment as disease control strategy to optimize the objective function $\mathrm{J}$.

Figure 8 (a), (b) and (c), represents the numbers of infected non-quarantined $I_{h}$, hospitalized (infected isolated) $H_{p}$ and infected mosquitoes $I_{v}$. From the figure, it is clearly seen that the numbers of infected nonquarantined humans and hospitalized (infected isolated) are decreased more with time incase with control than without control but their number cannot be zero at the final time of the implementation of the strategy. Similarly, the number of infected mosquitoes are large incase without control while their numbers are small incase with control at final time of implementation of the strategy. The reason is that applying optimized the combination of indoor residual spray IRS, screening and treatment only control intervention decreases more the burden of the disease than a combination of two controls intervention but it cannot be eradicate the disease in the community.

\subsection{Control with Insecticide Treated Net ITN, Treatment and Indoor Residual Spray IRS}

In this strategy, we applied a combination of treatment, Insecticide Treated Net ITN and Indoor Residual Spray IRS to the endemic malaria disease model system (1) as control strategy. In Figure 9 (a), (b) and (c) a small number difference is seen between states with control $\left(u_{1}(t) \neq 0, u_{3}(t) \neq 0 . u_{4}(t) \neq 0, u_{2}(t)=0\right)$ and without controls $\left(u_{1}(t)=u_{2}(t)=u_{3}(t)=u_{4}(t)=0\right)$. It is clearly seen from the figure that, the number of infected non-quarantined humans, hospitalized (infected isolated) humans and infected mosquitoes are decreased more with time incase with control than without control 
but their number cannot be zero at the final time of the implementation of the strategy. The reason is that applying optimized the combination of ITN, treatment, and IRS only control intervention, decreases more the burden of the disease than a combination of the two controls intervention but it cannot be eradicate the disease in the community.

\subsection{Control with Insecticide Treated Net ITN, Screening and Indoor Residual Spray IRS}

In this strategy, we applied a combination of screening, insecticide treated net ITN and indoor residual spray IRS to the endemic malaria disease model system (1) as control strategy.
In Figure 10 (a), (b) a small number difference is seen between states with control $\left(u_{1}(t) \neq 0, u_{2}(t) \neq 0 . u_{4}(t) \neq\right.$ $\left.0, u_{3}(t)=0\right)$ represented by blue color and without controls $\left(u_{1}(t)=u_{2}(t)=u_{3}(t)=u_{4}(t)=0\right)$ represented by red color. It is clearly seen from the figure that, the number of infected non-quarantined humans and infected mosquitoes are decreased more with time incase with control than without control but their number cannot be zero at the final time of the implementation of the strategy. The reason is that applying optimized the combination of ITN, screening, and IRS only control intervention, decreases more the burden of the disease than a combination of the two controls intervention but it cannot be eradicate the disease in the community.

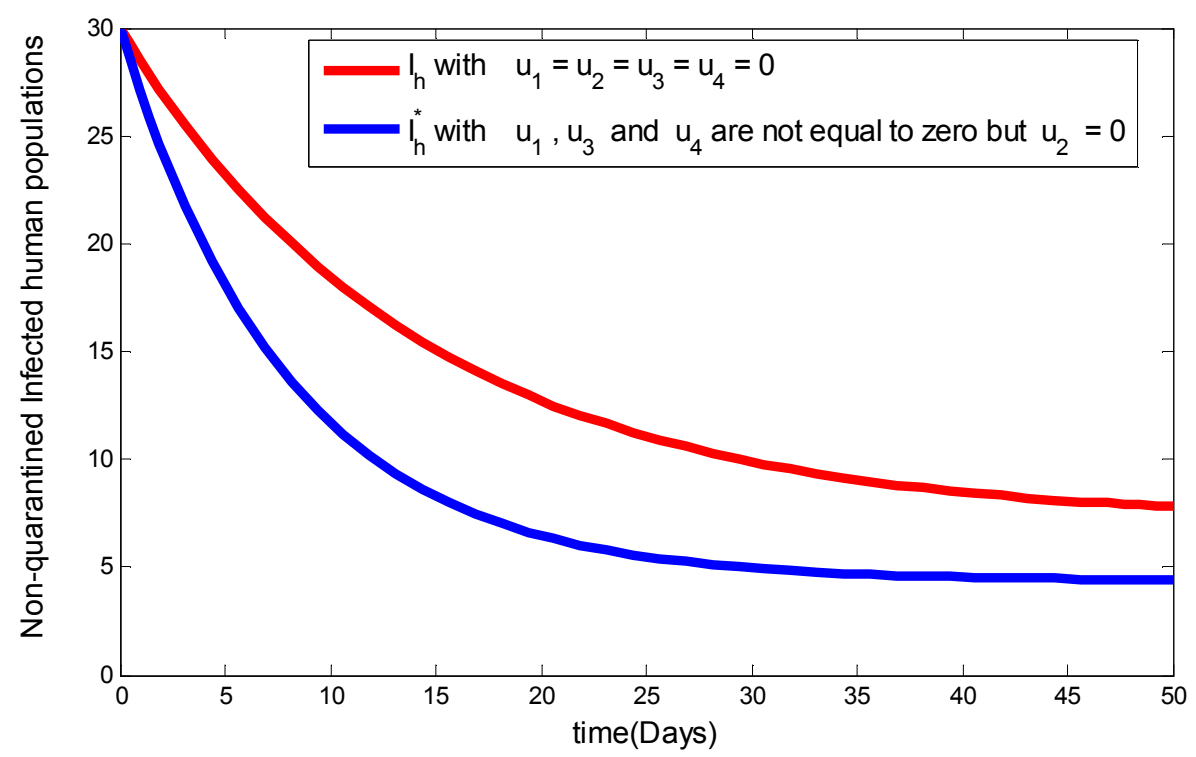

(a)

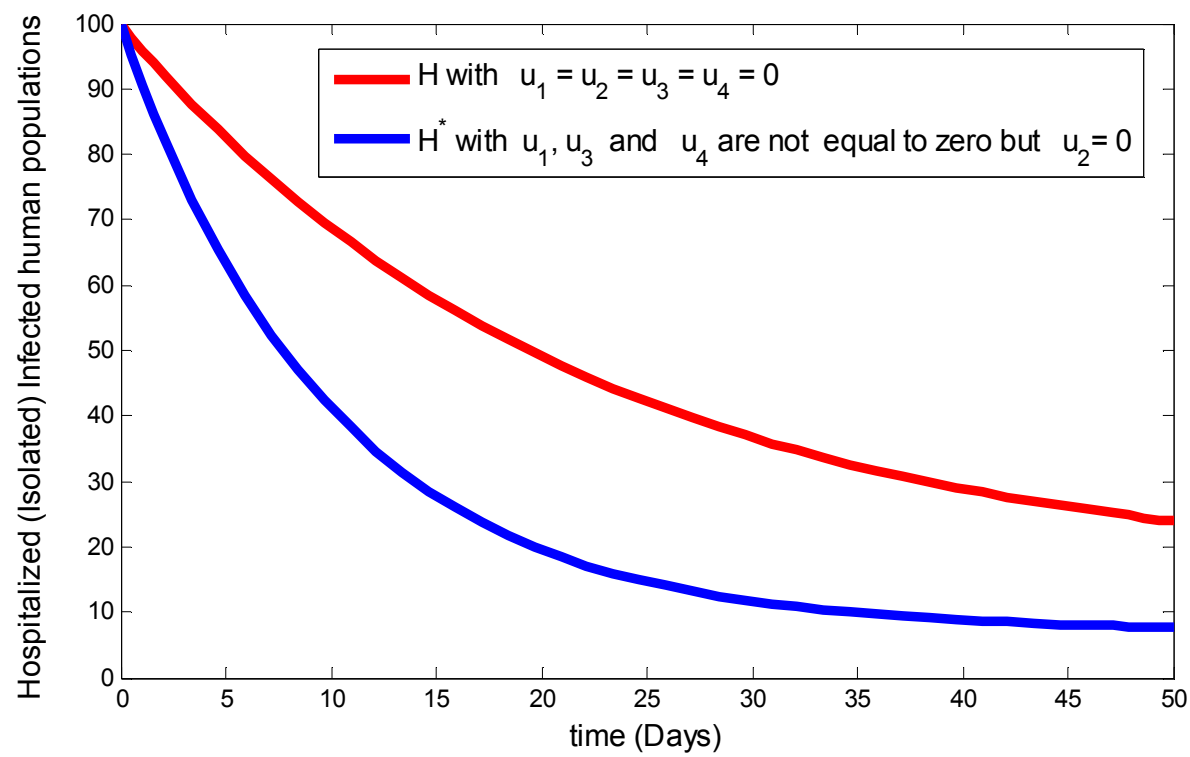

(b) 

Imperfect Quarantine and Optimal Control

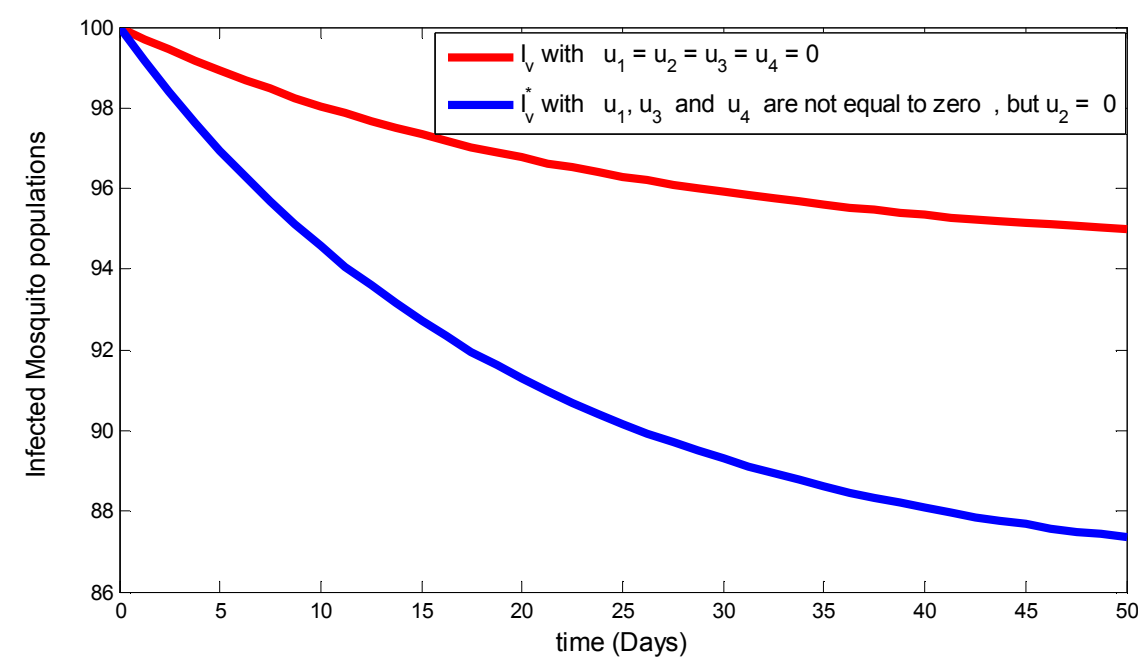

(c)

Figure 9. Simulation of endemic malaria model with Insecticide Treated Net, treatment and IRS.

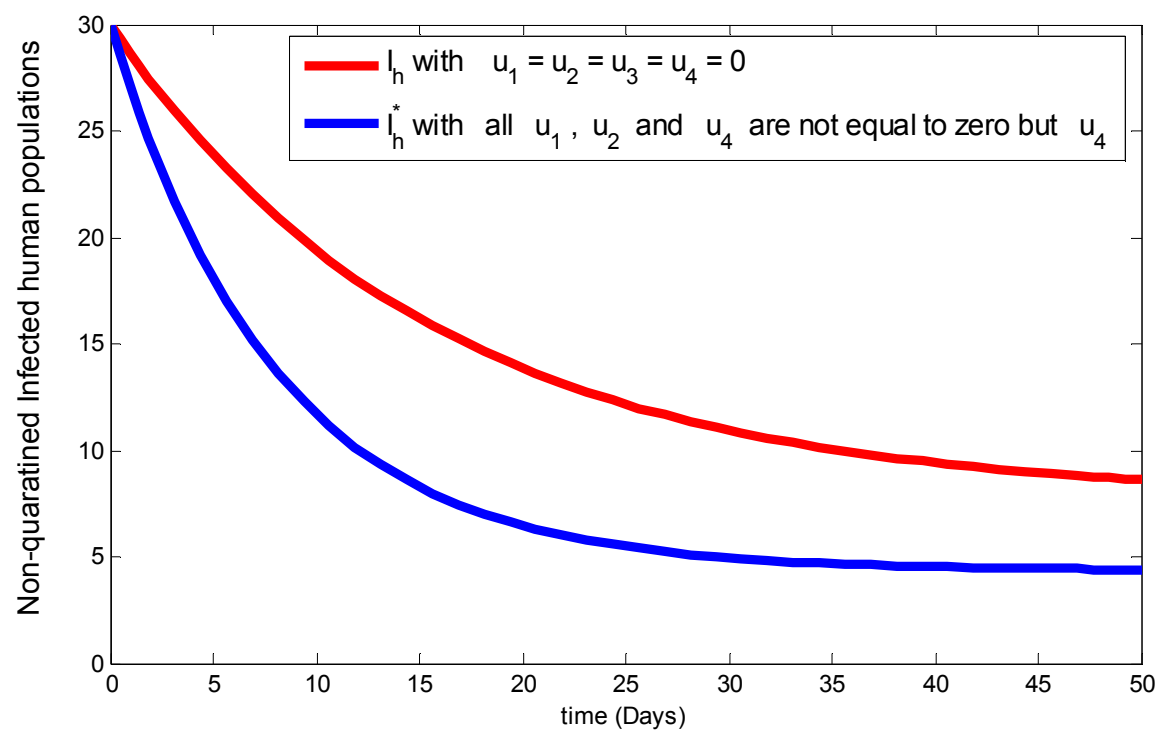

(a)

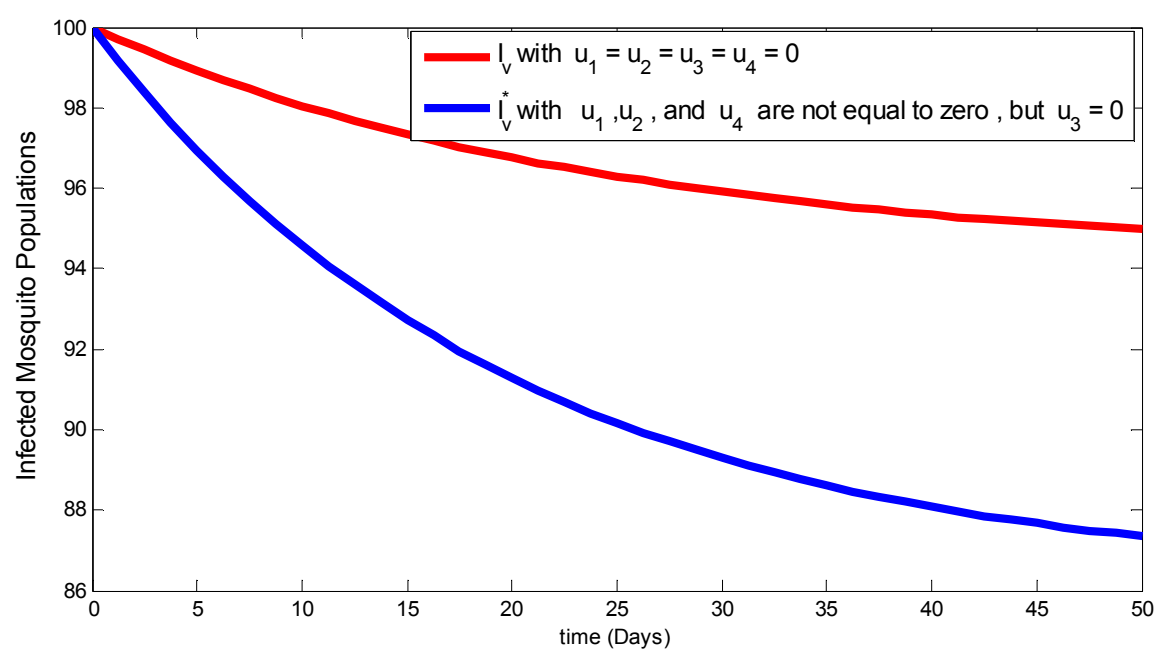

(b)

Figure 10. Simulation of endemic malaria model with Screening, ITN and IRS. 


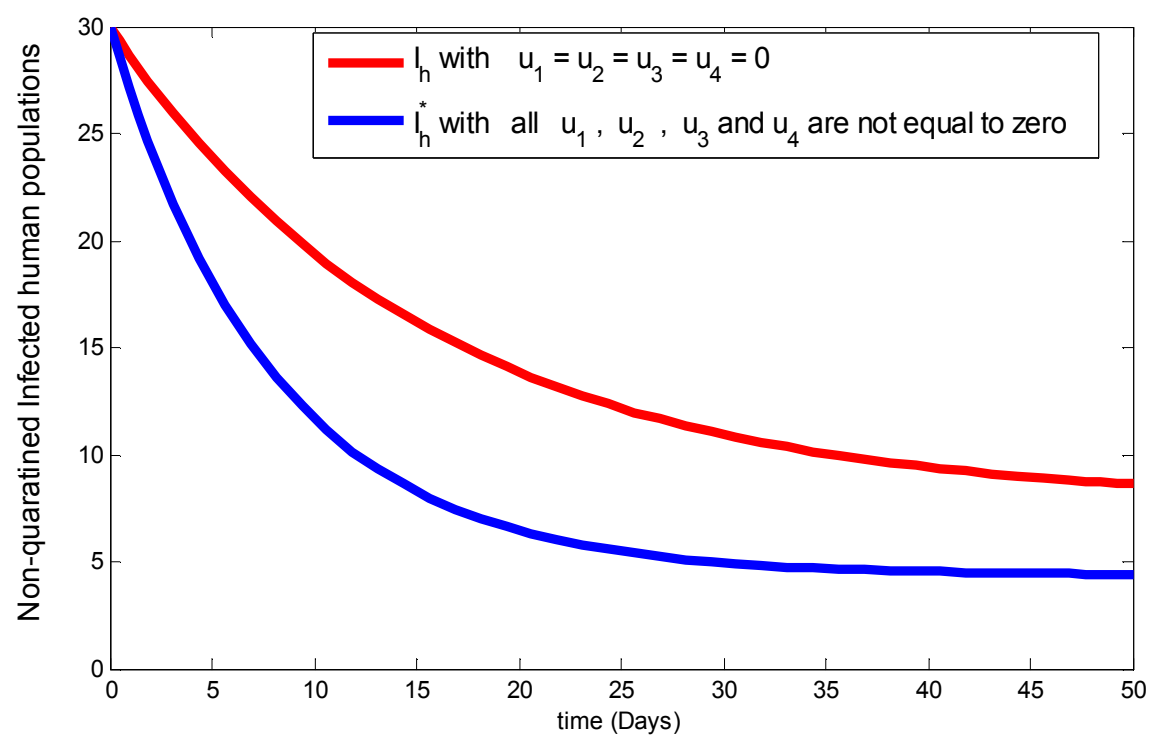

(a)

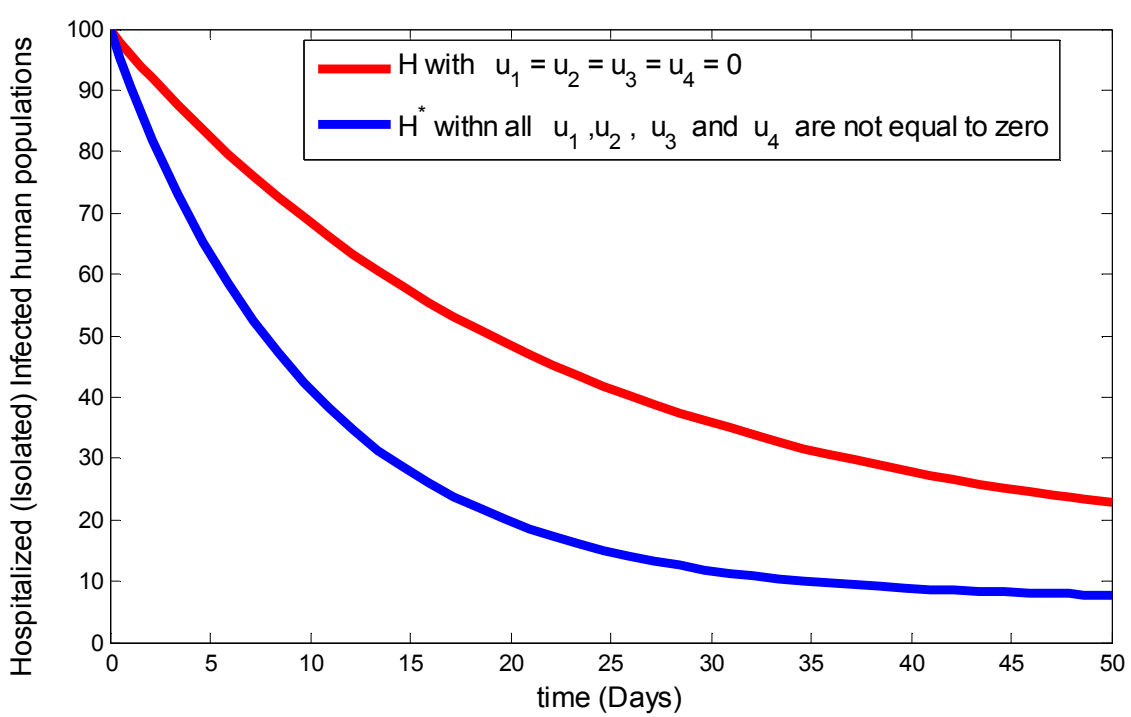

(b)

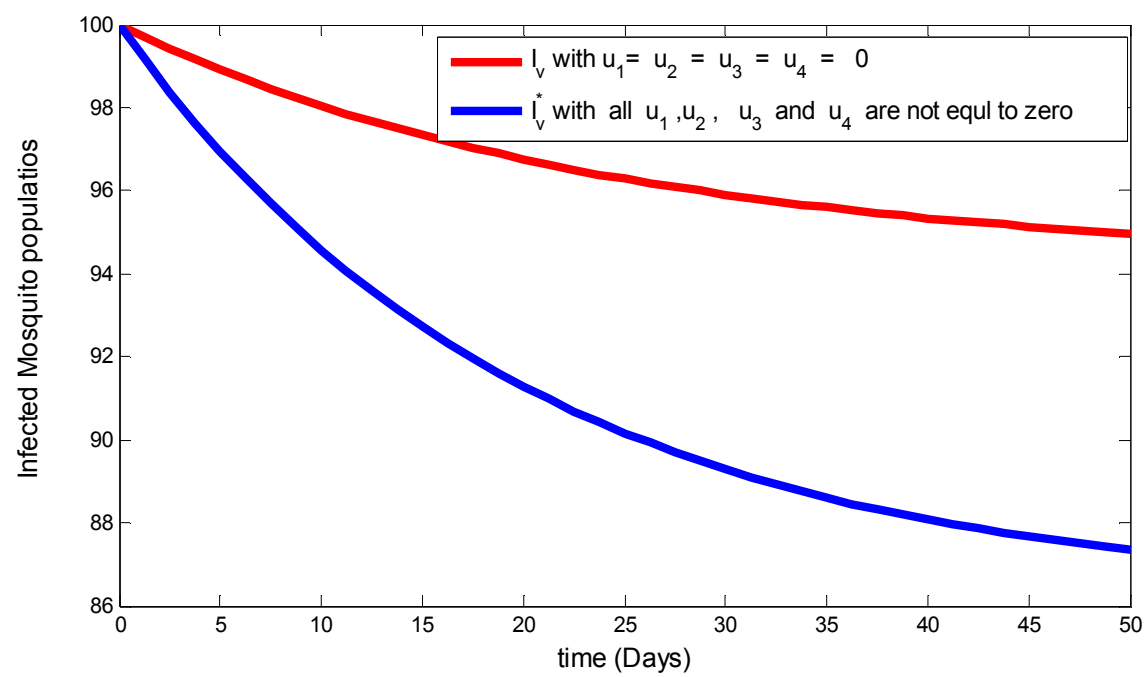

(c)

Figure 11. Simulation of endemic malaria model with screening, treatment, ITN and IRS. 


\subsection{Control with Screening, Treatment with Drugs, Insecticide Treated Net and Indoor Residual Spray}

In this strategy, we applied a combination of screening, treatment, Insecticide Treated Net ITN and Indoor Residual Spray IRS to the endemic malaria disease model system (1) as control strategy.

In Figure 11 (a), (b) and (c) above, a small number difference is seen between states with control $\left(u_{1}(t) \neq\right.$ $\left.0, u_{2}(t) \neq 0 . u_{3}(t) \neq 0, u_{4}(t) \neq 0\right)$ and without controls $\left(u_{1}(t)=u_{2}(t)=u_{3}(t)=u_{4}(t)=0\right)$. It is clearly seen from the figure that, the number of infected non-quarantined humans, hospitalized (infected isolated) humans and infected mosquitoes are exponentially more decreased with time incase with control than without control but their number cannot be zero at the final time of the implementation of the strategy. The reason is that applying optimized the combination of ITN, screening, treatment and IRS only control intervention, decreases furthermore the burden of the disease than a combination of the three controls intervention but it cannot be eradicate the disease in the community.

\section{Discussion and Conclusion}

In this paper, we formulated and analyzed a deterministic model that incorporates both imperfect quarantine and optimal control strategy to investigate their roles in case of endemic malaria disease control and elimination. We analyzed the dynamical behavior of the model in term of the basic reproduction number $R_{0}$ and also obtained a sufficient condition for both local and global asymptotic stability of the disease-free equilibrium $E_{0}$ and local asymptotic stability of endemic equilibrium $E_{* *}$. The model system (1) exhibit both backward and forward bifurcations at $R_{0}=1$.

The impact of imperfect quarantine strategy on endemic malaria persistence clearly seen on Figure 3 (figure showing Susceptible mosquitoes with and with no control parameter $\sigma)$. From this we conclude that in order to minimize the burden of malaria disease from the community, reducing the biting rate of the quarantined people is advice able than to quarantine more infected people at earlier infection stage.

The optimal control includes the use of insecticide treated nets, screening of infectious humans, treatment of infective humans and indoor residual spray to reduce the number of malaria transmitter vectors by means of spraying on the place where they choose for rest and breed. We perform and analyzed the necessary conditions for the optimal control of the disease model system (1). From this we conclude that,

(i) a combination of insecticide treated net and indoor residual spray is the best alternative combination of controls to reduce the numbers of infected nonquarantined humans and mosquitoes, when combinations of bi-controls are considered.

(ii) Both combinations of insecticide treated net-indoor residual spray- screening and insecticide treated netindoor residual spray treatment are the best alternative combinations of controls to reduce the numbers of infected non-quarantined humans, isolated humans and mosquitoes, when combinations of tri-controls are considered.

(iii)Furthermore, the best combination is the one that incorporated all four control strategies.

\section{Acknowledgements}

The authors would like to say thanks to the editors and reviewers of journal of MMA for their good suggestions and comments to this paper.

\section{References}

[1] Brooker S, Akhwale W, Pullan R, Estambale B, Clarke SE, Snow RW, et al. Epidemiology ofPlasmodium-helminth coinfection in Africa: populations at risk, potential impact on anemia, and prospects for combining control. Am J Trop Med Hyg. 2007; 77: 88-98.

[2] Marsh K, et al. Indicators of life-threatening malaria Africa children. N. Eng. J. Med. 1995; 332 (21): 1399-1404.

[3] Babikera HA, et al. The role of asymptomatic P. falciparum parasitaemia in the evoluation of antimalarial drug resistance in areas of seasonal transmission. Drug Resist Updates. 2013; $16(2): 1-9$.

[4] World malaria report 2017. Available: http://www.who.int/malaria/publications /world-malariareport-2017/report/en/. Accessed date: 15.8.2018.

[5] WorldMalaria Report $2015 . \quad$ Available: http://www.who.int/malaria/publications /world-malariareport-2015/report/en/. Accessed date: 20.7.2018.

[6] Olaniyi S, Obabiyi OS. Mathematical model for malaria transmission dynamics in human and mosquito populations with nonlinear forces of infection. International Journal of Pure and Applied Mathematics. 2013; 88 (1): 125-156.

[7] Ross R. The prevention of malaria, John Murray, London; 1911.

[8] Alemu Geleta Wedajo, Boka Kumsa Bole, Purnachandra Rao Koya. The Impact of Susceptible human Immigrants on the Spread and Dynamics of Malaria Transmission. American Journal of Applied Mathematics. Vol. 6, No. 3, 2018, pp. 117 - 127. Doi: 10.11648/j.ajam.20180,603.13.

[9] Alemu Geleta Wedajo, Boka Kumsa Bole, Purnachandra Rao Koya. Analysis of SIR Mathematical Model for Malaria disease with the inclusion of Infected Immigrants. IOSR Journal of Mathematics (IOSR - JM), Vol 14, Issue 5, Ver. I, Sep - Oct 2018, Pp 10 - 21. DOI: 10.9790/5728-1405011021.

[10] Tumwiine J., Mugisha J., Luboobi L., “A mathematical model for the dynamics of malaria in a human host and mosquito vector with temporary immunity", Journal of Applied Mathematics and Computation, vol. 189, pp. 1953-1965, 2005.

[11] Yang H., Wei H., Li X., "Global stability of an epidemic model for vector borne disease", J Syst Sci Complex Journal, vol. 23, pp. 279-292, 2010. 
[12] Feng Z, Thieme HR Recurrent outbreaks of childhood diseases revisited: the impact of isolation. Math Biosci 128 (2): 93-130, 1995.

[13] Hethcote HW, Ma Z, Liao S Effects of quarantine in six endemic models for infectious diseases. Math Biosci 180 (1): 141-160, 2002.

[14] Gumel AB, Ruan S, Day T, Watmough J, Brauer F, van den Driessche P, Gabrielson D, Bowman C, Alexander ME, Ardal $\mathrm{S}, \mathrm{Wu}$ J, Sahai BM Modeling strategies for controlling SARS outbreaks. Proc R Soc Lond B 271 (1554): 2223-2232, 2004.

[15] Pandey A, Atkins KE, Medlock J, Wenzel N, Townsend JP, Childs JE, Nyenswah TG, Ndeffo-Mbah ML, Galvani AP Strategies for containing Ebola in west Africa. Science 346 (6212): 991-995, 2014.

[16] Erdem M, Safan M, Castillo-Chavez C Mathematical analysis of an SIQR influenza model with imperfect quarantine. Bull Math Biol 79 (7): 1612-1636, 2017.

[17] Xiulei Jin, Shuwan Jin and Daozhou Gao, mathematical analysis of the Ross-Macdonald SIQ-SI malaria model with quarantine. Bulletin of Mathematical Biology 82: 47, 2020.

[18] K. O. Okosun et al. Malaria disease transmission mathematical model that incorporate optimal control strategy which includes insecticide treated net, treatment and indoor residual spray 2013.

[19] Suresh formulated and analyzed an optimal control problem with a simple epidemic model to examine effect of a quarantine program 1978.

[20] Otieno, G.; Koske, J. K.; Mutiso, J. M. A Model for Malaria Transmission Dynamics with Interventions Strategies in Kenya 2015.

[21] O. Diekmann, J. A. P. Heesterbeek and J. A. J. Metz, on the definition and computation of the basic reproduction ratio in models for infectious diseases in heterogeneous populations, J. Math. Biol, 28 (1990), 365-382.

[22] P. van den Driessche and J. Watmough, "Reproduction numbers and sub-threshold endemic equilibria for compartmental models of disease transmission," Mathematical Biosciences, vol. 180, pp. 29-48, 2002.

[23] C. Castillo-Chavez, Z. Feng and W. Huang, On the Computation of $R_{0}$ and Its Role on Globbal Stability. IMA Vol. Math-Appl. Sprenger, New York, 2002.

[24] Castillo-Chavez, C., Song, B.: Dynamical models of tuberculosis and their applications. Math. Biosci.

[25] van den Driessche, P., Watmough, J.: Reproduction numbers and sub-threshold endemic equilibria for compartmental models of disease transmission. Math. Biosci. 180, 29-48 (2002).

[26] Dushoff, J., Huang, W., Castillo-Chavez, C.: Backward bifurcations and catastrophe in simple models of fatal diseases. J. Math. Biol. 36, 227-248 (1998).

[27] Guckenheimer, J., Holmes, P.: Nonlinear Oscillations, Dynamical Systems, and Bifurcations of VectorFields, Applied Mathematical Sciences, vol. 42, 7 edn. Springer-Verlag, New York (2002).

[28] S. Baba and O. D. Makinde, "Optimal control of HIV/AIDS in the workplace in the presence of careless individuals," Computational and Mathematical Methods in Medicine, vol. 2014, Article ID 831506, 2014.

[29] Otieno, G.; Koske, J. K.; Mutiso, J. M. Optimal Control Strategies for the Spread of Malaria in Kenya. 2015, submitted.

[30] Fleming, W. H.; Rishel, R. W. Deterministic and Stochastic Optimal Control; Springer Verlag: New York, NY, USA, 1975.

[31] S. M. Lenhart and J. T. Workman, Optimal Control Applied to Biological Models, CRC Press, 2007.

[32] Otieno, G.; Koske, J. K.; Mutiso, J. M. Transmission Dynamics and Optimal of malaria in Kenya, H. P. C. Discrete Dynamics in Nature and Society 2016.

[33] KNBS, Kenya Population and Housing Census 2009, National Development and Vision 2030, KNBS, Ministry of Planning, Nairobi, Kenya, 2010.

[34] KNBS and ICF Macro, Kenya Demographic and Health Survey, 2008-2009, Kenya National Bureau of Statistics (KNBS) and ICF Macro, Calverton, Md, USA, 2010.

[35] C. Chiyaka, J. M. Tchuenche, W. Garira, and S. A. Dube, "A mathematical analysis of the effects of control strategies on the transmission dynamics of malaria," Applied Mathematics and Computation, vol. 195, no. 2, pp. 641-662, 2008.

[36] K. Blayneh, Y. Cao, and H.-D. Kwon, "Optimal control of vector-borne diseases: treatment and prevention," Discrete and Continuous Dynamical Systems. Series B, vol. 11, no. 3, pp. 587-611, 2009.

[37] Flahault, A.; Le Menach, A.; McKenzie, E. F.; Smith, D. L.; The unexpected importance of mosquito oviposition behavior for malaria: non-productive larval habitats can be sources for malaria transmissionn. Malaria Journal (2005).

[38] Chiyaka, C.; Tchuenche, J. M.; Garira, W.; Dube, S.; A Mathematical analysis of the effects of control strategies on the transmission dynamics of Malaria. Applied Mathematics and Computation, 195 (2008), 641-662.

[39] Smith, R. J.; Hove-Musekwa, S. D.; Determining effective spraying periods to control malaria via indoor residual spraying in sub-saharan Africa. J. Applied Mathematics and Decision Sciences. Article ID 745463, (2008). 